T. M. Gendron

Nagoya Math. J.

Vol. 190 (2008), 1-34

\title{
THE GEOMETRIC THEORY OF THE FUNDAMENTAL GERM
}

\author{
T. M. GENDRON
}

\begin{abstract}
The fundamental germ is a generalization of $\pi_{1}$, first defined for laminations which arise through group actions [4]. In this paper, the fundamental germ is extended to any lamination having a dense leaf admitting a smooth structure. In addition, an amplification of the fundamental germ called the mother germ is constructed, which is, unlike the fundamental germ, a topological invariant. The fundamental germs of the antenna lamination and the $P S L(2, \mathbb{Z})$ lamination are calculated, laminations for which the definition in [4] was not available. The mother germ is used to give a new proof of a Nielsen theorem for the algebraic universal cover of a closed surface of hyperbolic type.
\end{abstract}

\section{$\S 1$. Introduction}

This paper represents a continuation of our quest to extend $\mathbb{Z}$-coefficient algebraic topology to laminations through the generalization of $\pi_{1}$ called the fundamental germ. In this paper, we extend this construction to any lamination admitting a smooth structure.

Let us recall briefly the intuition behind the fundamental germ. Consider a suspension

$$
\mathscr{L}_{\rho}=(\widetilde{B} \times \mathrm{T}) / \pi_{1} B
$$

of a representation $\rho: \pi_{1} B \rightarrow \operatorname{Homeo}(\mathrm{T})$, where $B$ is a manifold. Then $\pi_{1} B$ acts on $\mathscr{L}_{\rho}$ as fiber preserving homeomorphisms. Let $T \approx \mathrm{T}$ be a fiber transversal and let $x_{0}, x, \in T$. A $\pi_{1} B$-diophantine approximation of $x \in T$ based at $x_{0}$ is a sequence $\left\{g_{\alpha}\right\} \subset \pi_{1} B$ with $g_{\alpha} \cdot x_{0} \rightarrow x$. The fundamental germ $\llbracket \pi \rrbracket_{1}\left(\mathscr{L}, x_{0}, x\right)$ is then the groupoid of tail equivalence classes of sequences of the form $\left\{g_{\alpha} \cdot h_{\alpha}^{-1}\right\}$ where $\left\{g_{\alpha}\right\},\left\{h_{\alpha}\right\}$ are diophantine approximations of $x$ along $x_{0}$ [4]. This construction is more generally available for any lamination occurring as a quotient of a suspension, a double-coset of a Lie group or a locally-free action of a Lie group on a space, laminations

Received August 4, 2005.

2000 Mathematics Subject Classification: Primary 57R30. 
which we refer to collectively as algebraic. Intuitively, if $L$ is the leaf containing $x_{0}$, the elements of $\llbracket \pi \rrbracket_{1}\left(\mathscr{L}, x_{0}, x\right)$ can be thought of as sequences of paths in $L$ whose endpoints converge transversally to $x$. Such a sequence can be thought of as an ideal loop based at $x$ that records an "asymptotic identification" within the leaf $L$.

For a linear foliation $\mathscr{F}_{r}$ of a torus by lines of slope $r$, the diophantine analogy is literal and $\llbracket \pi \rrbracket_{1}\left(\mathscr{F}, x_{0}, x\right)$ is the group of classical diophantine approximations of $r$. A manifold $B$ is a supension of the trivial representation i.e. a lamination with a single leaf and fiber transversals that are points, which forces $x_{0}=x$. Then all sequences in $\pi_{1} B$ converge, and we find that $\llbracket \pi \rrbracket_{1}(B, x)={ }^{*} \pi_{1}(B, x)=$ the nonstandard fundamental group of $B$.

We now turn to the contents of this article. The algebraic definition of the fundamental germ just described, while amenable to calculation, has the following serious drawbacks:

(1) It is available only for the select family of algebraic laminations.

(2) It is an invariant only with respect to the special class of trained lamination homeomorphisms ( $c f .[4])$.

Addressing these flaws is the central theme of the present study. In the summary that follows, we shall assume for simplicity that all leaves are simply connected.

We begin with item (1). Let $\mathscr{L}$ be an arbitrary lamination admitting a smooth structure, let $x_{0}, x$ be as above and denote by $L$ the leaf containing $x_{0}$. Equip $\mathscr{L}$ with a leaf-wise riemannian metric that has continuous transverse variation. In this paper, we shall refer to such a lamination as riemannian. The new idea here is to use the leaf-wise geometry to represent - as sequences of isometries - the diophantine approximations which would make up $\llbracket \pi \rrbracket_{1}$. If $L$ has constant curvature geometry, this prescription may be followed word-for-word. Fixing a transversal $T$ containing $x_{0}$, $x$ and a continuous section of orthonormal frames $\mathrm{f}=\left\{\mathrm{f}_{y}\right\}, y \in T$, we define a diophantine approximation of $x$ to be a sequence $\left\{A_{\alpha}\right\}$ of isometries of $L$ for which $\left(A_{\alpha}\right)_{*} \mathrm{f}_{x_{0}}$ belongs to $\mathrm{f}$ and converges transversally to $\mathrm{f}_{x}$. The fundamental germ $\llbracket \pi \rrbracket_{1}\left(\mathscr{L}, x_{0}, x, \mathrm{f}\right)$ is then defined to be the set of tails of sequences of the form $\left\{A_{\alpha} B_{\alpha}^{-1}\right\}$ where $\left\{A_{\alpha}\right\},\left\{B_{\alpha}\right\}$ are diophantine approximations of $x$.

In the case of non constant curvature leaf-wise geometry, it is necessary to work within the category of virtual geometry in order to make sense of the notion of diophantine approximation. There, a riemannian manifold $M$ 
is replaced by a union of riemannian manifolds, its virtual extension $\bullet M$, which consists of all sequences in $M$ up to the relation of being asymptotic. A virtual isometry between riemannian manifolds $M$ and $N$ consists of a pair of isometric inclusions $\bullet M \leftrightarrows \bullet N$. All dense leaves of a riemannian lamination have virtually isometric universal covers, and moreover, a dense leaf having no ordinary isometries will admit many virtual isometries.

This leads to the following definition of a diophantine approximation: let $x \in T, \mathrm{f}$ a frame field on $T$ and let $L$ be any leaf accumulating on $x$. Then a sequence $\mathrm{f}_{x_{\alpha}} \rightarrow \mathrm{f}_{x},\left\{x_{\alpha}\right\} \subset L$, determines an isometry $\bullet f: L_{x} \rightarrow$ $U \subset \bullet L$, where $L_{x}$ is the leaf containing $x$ and $U$ is a component of $\bullet L$. The fundamental germ $\llbracket \pi \rrbracket_{1}(\mathscr{L}, L, x, \mathrm{f})$ is defined to be the set of (maximal extensions of) maps of the form

$$
\cdot f \circ g^{-1}
$$

In this way, we now have a definition of the fundamental germ valid for any lamination admitting a smooth structure along the leaves.

In order to address drawback (2), we will need the germ universal cover

$$
\llbracket \widetilde{\mathscr{L} \rrbracket} \subset \cdot L
$$

defined to be the set of asymptotic classes of sequences in $L$ that converge to points of $\mathscr{L}$. The germ universal cover plays the role of a unit space for a groupoid structure on $\llbracket \pi \rrbracket_{1}(\mathscr{L}, L, x, \mathrm{f})$. It is a lamination whose leaves are nowhere dense, and when $L$ is dense, the canonical map $\llbracket \widetilde{\mathscr{L}} \rrbracket \rightarrow \mathscr{L}$ is onto. We may therefore think of $\llbracket \widetilde{\mathscr{L} \rrbracket}$ as obtained from $\mathscr{L}$ by "unwrapping" all transversal topology implemented by $L$.

Assume now that $L$ is dense. The mother germ $\llbracket \pi \rrbracket_{1} \mathscr{L}$ is defined to be the groupoid of all partially defined maps of $\llbracket \widetilde{\mathscr{L}} \rrbracket$ that are homeomorphisms on domains which are sublaminations of $\llbracket \widetilde{\mathscr{L}} \rrbracket$ and preserve the projection $\llbracket \widetilde{\mathscr{L}} \rightarrow \rightarrow \mathscr{L}$. We have in particular that

$$
\llbracket \pi \rrbracket_{1} \mathscr{L} \backslash \llbracket \widetilde{\mathscr{L}} \rrbracket \cong \mathscr{L}
$$

We shall see that $\llbracket \pi \rrbracket_{1} \mathscr{L}$ is the receptacle of all the $\llbracket \pi \rrbracket_{1}\left(\mathscr{L}, L^{\prime}, x, \mathrm{f}\right)$ for $L^{\prime}$ dense, in that it contains subgroupoids isomorphic to each. The mother germ is functorial with respect to topological lamination covering maps, and is therefore, in spite of its riemannian construction, a topological invariant. This takes care of item (2) above. 
The remainder of the paper is devoted to examples and an application. Many examples were discussed in [4], and so for this reason we limit ourselves to laminations which are not algebraic and hence which do not have a fundamental germ in the sense described there.

The first example we consider is that which we call here the antenna lamination, a surface lamination discovered by Kenyon and Ghys [6] which has the distinction of having leaves of both parabolic and hyperbolic type. With respect to a hyperbolic leaf, the fundamental germ is calculated as a set to be ${ }^{*} F_{2} \times\left({ }^{*} \mathbb{Z}_{\hat{2}} \oplus^{*} \mathbb{Z}_{\hat{2}}\right)$ where ${ }^{*} F_{2}$ is the nonstandard free group on two generators, and $* \mathbb{Z}_{\hat{2}}$ is the subgroup of $* \mathbb{Z}$ isomorphic to the fundamental germ of the dyadic solenoid. Although a product of groups, this germ is not a group with respect to its defined multiplication.

The second example is that of the Anosov foliation of the unit tangent bundle to the modular surface. Although this is just the suspension of the action of $P S L(2, \mathbb{Z})$ on the boundary of the hyperbolic plane, the definition of the fundamental germ found in [4] is unavailable since it does not work for actions with fixed points. We calculate the fundamental germ here as a set to be $P S L\left(2,{ }^{*} \mathbb{Z}\right)$, but as in the case of the antenna lamination, it is also not a group with respect to its defined multiplication.

The final result of this paper concerns the use of the fundamental germ to calculate the mapping class group of the algebraic universal cover $\widehat{\Sigma}$ of a closed surface $\Sigma$ of hyperbolic type. $\widehat{\Sigma}$ is by definition the inverse limit of finite covers of $\Sigma$, a compact solenoid with dense disk leaves. If $L \subset \widehat{\Sigma}$ is a fixed leaf, the leafed mapping class group $\operatorname{MCG}(\mathscr{L}, L)$ is the quotient $\mathrm{Homeo}_{+}(\mathscr{L}, L) / \simeq$, where $\mathrm{Homeo}_{+}(\mathscr{L}, L)$ denotes the group of orientationpreserving homeomorphisms fixing set-wise $L$ and $\simeq$ denotes homotopy. If we denote by $\operatorname{Vaut}\left(\pi_{1} \Sigma\right)$ the group of virtual automorphisms of $\pi_{1} \Sigma(c f$. Section 10) then

THEOREM. There is an isomorphism

$$
\Theta: \operatorname{MCG}(\mathscr{L}, L) \longrightarrow \operatorname{Vaut}\left(\pi_{1} \Sigma\right)
$$

This theorem - which is an important ingredient in the genus-independent expression of the Ehrenpreis conjecture [5] - was first proved in [8]. We will give a new proof which uses the mother germ and germ covering space theory. 
Acknowledgements. I would like to thank P. Makienko and A. Verjovsky with whom I enjoyed fruitful conversations regarding several important aspects of this paper. I would also like to thank the Instituto de Matemáticas of the UNAM for providing a pleasant work enviroment and generous financial support.

\section{§2. Virtual geometry}

Virtual geometry is obtained as a quotient of nonstandard geometry, which we now review: references [7], [9], [10].

Let $M$ be a topological space, $\mathfrak{U} \subset 2^{\mathbb{N}}$ an ultrafilter on the natural numbers all of whose elements have infinite cardinality. The nonstandard space ${ }^{*} M$ is the set of sequences in $M$ modulo $\mathfrak{U}$ : that is,

$$
\left\{x_{i}\right\} \sim\left\{y_{i}\right\} \text { if and only if }\left.\left\{x_{i}\right\}\right|_{X}=\left.\left\{y_{i}\right\}\right|_{X} \text { for some } X \in \mathfrak{U}
$$

Elements of ${ }^{*} M$ are denoted ${ }^{*} x$. There is a natural map $M \hookrightarrow{ }^{*} M$ given by the constant sequences. Modulo the continuum hypothesis, ${ }^{*} M$ is independent of the choice of ultrafilter.

There are two topologies on ${ }^{*} M$ that naturally suggest themselves. The enlargement topology is generated by sets of the form ${ }^{*} O$, where $O$ is open in $M$. It has the same countability as the topology of $M$ but is non-Hausdorff. The internal topology is generated by sets of the form $\left[O_{\alpha}\right]=\left\{\left.{ }^{*} x \in{ }^{*} M\right|^{*} x\right.$ is represented by a sequence $\left.\left\{x_{\alpha}\right\}, x_{\alpha} \in O_{\alpha}\right\}$, where $\left\{O_{\alpha}\right\}$ is any sequence of open sets of $M$. It is Hausdorff but has greater countability than the topology of $M$.

If $f: M \rightarrow N$ is continuous, the nonstandard extension ${ }^{*} f:{ }^{*} M \rightarrow{ }^{*} N-$ defined ${ }^{*} f\left({ }^{*} x\right)=$ the equivalence class of $\left\{f\left(x_{i}\right)\right\}$ for $\left\{x_{i}\right\}$ any representative of ${ }^{*} x$ - is continuous with respect to the enlargement (internal) topology on ${ }^{*} M,{ }^{*} N$.

For example, if we let $M=\mathbb{R}$ we obtain the nonstandard reals $* \mathbb{R}$, a totally ordered, non-archemidean field. Note that $* \mathbb{R}$ is an infinite-dimensional vector space over $\mathbb{R}$. We will refer to the following substructures of the nonstandard reals:

- The subring of bounded nonstandard reals, denoted ${ }^{*} \mathbb{R}_{\mathrm{fin}}$, which consists of all classes of sequences that are bounded.

- The additive subgroup of infinitesimals, denoted $* \mathbb{R}_{\epsilon}$, which consists of all classes of sequences converging to 0 . 
- The cone of positive elements, denoted ${ }^{*} \mathbb{R}_{+}$, which consists of all classes of sequences that are $\geq 0$.

Note that ${ }^{*} \mathbb{R}_{\text {fin }}$ is a local topological ring in either the enlargement or internal topology, with maximal ideal ${ }^{*} \mathbb{R}_{\epsilon}$. The quotient ${ }^{*} \mathbb{R}_{\mathrm{fin}} /{ }^{*} \mathbb{R}_{\epsilon}$ is isomorphic to $\mathbb{R}$, homeomorphic with the quotient enlargement topology (the quotient internal topology is discrete). The inclusion $\mathbb{R} \hookrightarrow{ }^{*} \mathbb{R}_{\text {fin }}$ allows us to canonically identify ${ }^{*} \mathbb{R}_{\text {fin }}$ with the product $\mathbb{R} \times{ }^{*} \mathbb{R}_{\epsilon}$. Taking the product of the euclidean topology on $\mathbb{R}$ with the discrete topology on ${ }^{*} \mathbb{R}_{\epsilon}$, we obtain a third topology on ${ }^{*} \mathbb{R}_{\text {fin }}$ which is Hausdorff and quotients by ${ }^{*} \mathbb{R}_{\epsilon}$ to the topology on $\mathbb{R}$. We call this third topology the lamination topology: it may be extended to $* \mathbb{R}$ by giving the group $* \mathbb{R} / \mathbb{R}$ the discrete topology and identifying $* \mathbb{R} \cong \mathbb{R} \times\left({ }^{*} \mathbb{R} / \mathbb{R}\right)$.

If $M$ is an $n$-manifold, then ${ }^{*} M$ is a nonstandard manifold modelled on ${ }^{*} \mathbb{R}^{n}$. If we denote by ${ }^{*} M_{\text {fin }}$ the points of ${ }^{*} M$ represented by sequences which converge to points of $M$, then we may choose an atlas on ${ }^{*} M_{\text {fin }}$ whose transitions preserve the lamination structure of ${ }^{*} \mathbb{R}_{\text {fin }}^{n}$ i.e. ${ }^{*} M_{\text {fin }}$ is an $n$ lamination. Indeed, if $\{\phi\}$ is an atlas for the manifold structure of $M$, where the $\phi: U \subset M \rightarrow O \subset \mathbb{R}^{n}$ are assumed to be charts with relatively compact domains and ranges, then the collection $\left\{{ }^{*} \phi\right\}$ is such an atlas. In general, ${ }^{*} M$ is a union of laminations of dimensions $\leq n$, this because of the possibility of "dimension collapse" which we describe in the proof of Theorem 1 below.

If $d$ is a metric inducing the topology of $M$, it extends to a ${ }^{*} \mathbb{R}_{+}$-valued metric ${ }^{*} d$ on ${ }^{*} M$. Write ${ }^{*} x \simeq{ }^{*} x^{\prime}$ if ${ }^{*} d\left({ }^{*} x,{ }^{*} x^{\prime}\right) \in{ }^{*} \mathbb{R}_{\epsilon}$.

Definition 1. The virtual extension of $M$ is the quotient

$$
\cdot M={ }^{*} M / \simeq
$$

equipped with the quotient lamination topology.

The virtual extension of $\bullet \mathbb{R}$ of $\mathbb{R}$ is called the virtual reals, a totallyordered real vector space. The metric ${ }^{*} d$ on ${ }^{*} M$ induces a $\bullet \mathbb{R}_{+}$-valued metric $\cdot d$ on $\bullet M$. Given $\cdot x \in \bullet$, the set

$$
U_{\bullet}=\{\bullet y \mid \bullet d(\bullet x, \bullet y) \in \mathbb{R}\}
$$

is a component of $\bullet M$ called the galaxy of $\bullet$. Note that $M$ is a galaxy of $\bullet M$, and $\bullet M$ is the union of all of its galaxies. 
The galaxies of $\bullet M$ can be quite different from one another. For example if $M$ is simply connected, then there may be galaxies that are not simply connected. For example, suppose that $M$ is a noncompact leaf of the Reeb foliation, equipped with the metric induced from an embedding of the solid 2-torus in $\mathbb{R}^{3}$. Consider a sequence of points $\left\{x_{\alpha}\right\}$ in $M$ converging to a point $\hat{x}$ in the compact toral leaf. Let $\left\{\gamma_{\alpha}\right\}$ be a sequence of simple closed curves containing the sequence $\left\{x_{\alpha}\right\}$ and converging to the meridian through $\hat{x}$. Then the limit curve ${ }^{\bullet} \gamma$ is essential in the galaxy $U_{x}$. On the other hand, if $M$ is a riemannian homogeneous space, then the galaxies of $\bullet M$ are all isometric to $M$.

THEOREM 1. If $M$ is a complete riemannian manifold of dimension $n$, each galaxy $U$ of $\bullet M$ has the structure of a complete riemannian manifold of dimension $m \leq n$.

Proof. Given a galaxy $U,{ }^{\bullet} x \in U$ and $\left\{x_{\alpha}\right\}$ a representative sequence, let $m$ be the largest integer for which there exists a sequence of $m$-dimensional balls $\left\{D_{r}\left(x_{\alpha}\right)\right\}$ of fixed radius $r$ about $\left\{x_{\alpha}\right\}$. The integer $m$ is independent of the representative sequence and defines an $m$-ball $D_{r}(\bullet x) \subset U$. The function ${ }^{\bullet} x \mapsto m$ is locally constant, thus the collection of such balls defines on $U$ the structure of a smooth $m$-manifold. Note that it is possible to have $m<n$ : for example, if $M$ is a hyperbolic manifold with a cusp, then for a class of sequence emptying into the cusp, we have $m=n-1$.

Consider the nonstandard tangent bundle

$$
\mathbf{T}^{*} M:={ }^{*}(\mathbf{T} M)
$$

There is a natural projection of $\mathbf{T}^{*} M$ onto ${ }^{*} M$ whose fiber $\mathbf{T}^{*}{ }^{*} M-$ the tangent space at ${ }^{*} x$ - consists of classes of sequences of vectors $\left\{\mathrm{v}_{\alpha}\right\}$ based at sequences $\left\{x_{\alpha}\right\}$ belonging to the class of ${ }^{*} x$. It is not difficult to see that $\mathbf{T}_{*}{ }^{*} M$ is a real infinite-dimensional vector space. The riemannian metric $\rho$ extends to a ${ }^{*} \mathbb{R}$-valued metric ${ }^{*} \rho$ on $\mathbf{T}^{*} M$ in the obvious way. Denote by ${ }^{*}|\cdot|$ the associated norm. Define the bounded tangent bundle by

$$
\mathbf{T}_{\text {fin }}{ }^{*} M=\left\{{ }^{*} \mathrm{v} \in \mathbf{T}^{*} M\left|{ }^{*}\right|{ }^{*} \mathrm{v} \mid \in{ }^{*} \mathbb{R}_{\mathrm{fin}}\right\} .
$$

Given tangent vectors ${ }^{*} \mathrm{v}$ and ${ }^{*} \mathrm{v}^{\prime}$ based at ${ }^{*} x$ and ${ }^{*} x^{\prime}$, we write ${ }^{*} \mathrm{v} \simeq{ }^{*} \mathrm{v}^{\prime}$ if

(1) ${ }^{*} x \simeq{ }^{*} x^{\prime}$ 
(2) the Levi-Civita parallel translate of a representative $\left\{\mathrm{v}_{\alpha}\right\}$ of $* \mathrm{v}$ to a representative $\left\{x_{\alpha}^{\prime}\right\}$ of ${ }^{*} x^{\prime}$ - along a sequence of geodesics connecting to a representative $\left\{x_{\alpha}\right\}$ of ${ }^{*} x$ - is asymptotic to a representative $\left\{\mathrm{v}_{\alpha}^{\prime}\right\}$ of $* v^{\prime}$.

Now define the bounded tangent bundle of $\bullet M$ to be

$$
\mathbf{T}_{\text {fin }} \cdot M=\mathbf{T}_{\text {fin }}{ }^{*} M / \simeq
$$

The nonstandard riemannian metric ${ }^{*} \rho$ on $\mathbf{T}_{\mathrm{fin}}{ }^{*} M$ descends to a riemannian metric on $\mathbf{T}_{\text {fin }} \cdot M$. If $U$ is a galaxy, its tangent space may be identified with the restriction of $\mathbf{T}_{\text {fin }} \bullet M$ to $U$. Now any geodesic $\eta \subset U$ can be realized as a sequence class of geodesics $\left\{\eta_{\alpha}\right\}$. Since each member of such a sequence can be continued indefinitely, the same is true of $\eta$, hence $U$ is complete.

Definition 2. Let $M, N$ be riemannian $n$-manifolds. A virtual subisometry is an injective map

$$
\bullet f: \bullet \longleftrightarrow \cdot N
$$

where $\bullet$ maps each galaxy of $\bullet M$ isometrically onto a galaxy of $\bullet N$. If in addition there exists a virtual subisometry $\bullet g:{ }^{\prime} \hookrightarrow \hookrightarrow \bullet$, then the pair $(\bullet f, \bullet g)$ is called a virtual isometry.

We write $M \leq_{\text {vir }} N$ to indicate the existance of a virtual subisometry - $f$ and $M \cong_{\text {vir }} N$ indicates the existence of a virtual isometry. The relation $\leq_{\text {vir }}$ defines a partial ordering on the set of all riemannian $n$-manifolds.

An isometry $f: M \rightarrow N$ clearly induces a virtual isometry $(\bullet f, \bullet g)$ : $\bullet M \leftrightarrows \bullet N$ with $\bullet f, \bullet g$ inverse to one another. More generally, a continuous map $\bullet f: \bullet M \rightarrow N$ is called standard if it is induced by a map $f: M \rightarrow N$ i.e. if for any $\bullet \in \in \cdot M$ and any representative $\left\{x_{\alpha}\right\},\left\{f\left(x_{\alpha}\right)\right\}$ is a representative of $\bullet f(\bullet)$.

TheOREm 2. Let $L$ be a dense leaf of a riemannian lamination $\mathscr{L}$. Then for every leaf $L^{\prime} \subset \mathscr{L}$,

$$
\widetilde{L}^{\prime} \leq \text { vir } \widetilde{L}
$$

Proof. Fix a global metric $d$ on $\mathscr{L}$ which agrees locally with the riemannian metric on the leaves. (By this we mean that in sufficiently small 
flow boxes, $d$ agrees with the distance function of $\rho$ in any plaque.) Let $\left\{\tilde{x}_{\alpha}^{\prime}\right\} \subset \widetilde{L}^{\prime}$ be any sequence, $\left\{x_{\alpha}^{\prime}\right\}$ its projection to $L^{\prime}$. Let $\left\{\tilde{x}_{\alpha}\right\} \subset \widetilde{L}$ be a sequence whose projection $\left\{x_{\alpha}\right\}$ to $L$ is $d$-asymptotic to $\left\{x_{\alpha}^{\prime}\right\}$. By transversal continuity of the metric, we deduce a sequence of $K_{\alpha}$-quasiisometries, $K_{\alpha} \rightarrow 1$

$$
f_{\alpha}: D_{\delta}\left(x_{\alpha}\right) \longrightarrow D_{\delta}\left(x_{\alpha}^{\prime}\right),
$$

for some $\delta>0$, where $D_{\delta}(x)$ means the open $\rho$-ball of radius $\delta$ about $x$. Then if $\bullet \tilde{x}, \bullet \tilde{x}^{\prime}$ are the virtual classes of $\left\{\tilde{x}_{\alpha}\right\},\left\{\tilde{x}_{\alpha}^{\prime}\right\}$, the sequence of quasiisometries $\left\{f_{\alpha}\right\}$ induces an isometry $D_{\delta}(\bullet \tilde{x}) \rightarrow D_{\delta}\left(\bullet \tilde{x}^{\prime}\right)$. Since $L$ is dense, we may continue these isometries along geodesics to obtain a locally isometric surjection $U \rightarrow U^{\prime}$, where $U, U^{\prime}$ are the galaxies containing $\bullet \tilde{x}$, - $\tilde{x}^{\prime}$. But since these spaces are simply connected, and the map is isometric, this surjection is a bijection. Hence it inverts to an isometry $U^{\prime} \rightarrow U$. Repeating this for every ${ }^{\bullet}$-class of sequence in $\widetilde{L}^{\prime}$, we obtain the desired virtual subisometry $\widetilde{L}^{\prime} \leq$ vir $\widetilde{L}$.

Two riemannian manifolds have the same virtual geometry if their universal covers are virtually isometric.

Corollary 1. Dense leaves of a riemannian lamination $\mathscr{L}$ have the same virtual geometry.

\section{$\S 3 . ~ T h e ~ f u n d a m e n t a l$ germ}

Let $\mathscr{L}$ be a riemannian lamination, $x$ a point contained in a transversal $T, L$ a leaf accumulating at $x$ and $L_{x}$ the leaf containing $x$. Let $\mathrm{f}: T \rightarrow \mathbf{F}_{*} \mathscr{L}$ be a continuous section of the leaf-wise orthonormal frame bundle of $\mathscr{L}$ over $T$. Fix locally isometric universal covers $p: \widetilde{L} \rightarrow L$ and $p_{x}: \widetilde{L}_{x} \rightarrow L_{x}$. Denote $T_{0}=T \cap L, \widetilde{T}_{0}=p^{-1}\left(T_{0}\right)$ and let $\tilde{\mathrm{f}}_{\tilde{y}}$ denote the lift of $\mathrm{f}_{y}$ to a point $\tilde{y} \in \widetilde{T}_{0}$ covering $y$. We pick a basepoint $\tilde{x} \in \widetilde{L}_{x}$ lying over $x$ with lifted frame $\tilde{\mathrm{f}}_{\tilde{x}}$.

Let $\tilde{y} \in \widetilde{T}_{0}$. For $r>0$, the frames $\tilde{\mathrm{f}}_{\tilde{x}}, \tilde{\mathrm{f}}_{\tilde{y}}$ determine polar coordinates on the metric disks $D_{r}(\tilde{x}), D_{r}(\tilde{y})$. This yields in turn a canonical quasiisometry

$$
f: D_{r}(\tilde{x}) \longrightarrow D_{r}(\tilde{y})
$$

given by the coordinate maps.

Let $\left\{x_{\alpha}\right\} \subset T_{0}$ be a sequence converging to $x,\left\{\tilde{x}_{\alpha}\right\} \subset \widetilde{T}_{0}$ any sequence covering $\left\{x_{\alpha}\right\}$. Then the frame sequence $\left\{\tilde{\mathrm{f}}_{\tilde{x}_{\alpha}}\right\}$ and the frame $\tilde{f}_{\tilde{x}}$ determine 
a sequence of $K_{\alpha}$-quasiisometries

$$
\left\{f_{\alpha}: D_{r_{\alpha}}(\tilde{x}) \longrightarrow D_{r_{\alpha}}\left(\tilde{x}_{\alpha}\right)\right\}
$$

Since $L$ accumulates at $x$, we may choose the sequence of radii $r_{\alpha} \rightarrow \infty$ so that $K_{\alpha} \rightarrow 1$. We deduce an isometry

$$
\text { • } f: \widetilde{L}_{x} \longrightarrow U \subset \bullet \widetilde{L}
$$

where $U$ is the galaxy containing $\bullet \tilde{x}$. The map $\bullet f$ is called an $\mathbf{f}$-diophantine approximation of $x$ along $L$.

Definition 3. The fundamental germ of $\mathscr{L}$, based at $x$ along $L$ and $\mathrm{f}$, is

$$
\begin{array}{r}
\llbracket \pi \rrbracket_{1}(\mathscr{L}, L, x, \mathrm{f})=\left\{\bullet^{\circ} \circ \bullet^{-1} \mid \cdot f, \bullet g\right. \text { are f-diophantine approximations } \\
\text { of } x \text { along } L\} .
\end{array}
$$

If $x \in L$, we shorten the notation to $\llbracket \pi \rrbracket_{1}(\mathscr{L}, x, \mathrm{f})$. The groupoid structure of $\llbracket \pi \rrbracket_{1}(\mathscr{L}, L, x, \mathrm{f})$ will be described in the next section.

Note 1. Suppose that $\mathscr{L}$ is a constant curvature riemannian foliation with dense leaf $L$ modeled on the space form $\mathbb{M}^{n}=\mathbb{R}^{n}$ or $\mathbb{H}^{n}$. Then the frame field actually determines a sequence of uniquely defined global isometries $\left\{f_{\alpha}: \mathbb{M}^{n} \rightarrow \mathbb{M}^{n}\right\}$. Given $G$ a group, nonstandard $G$ is the group ${ }^{*} G$ of all sequences $\left\{g_{\alpha}\right\} \subset G$ modulo the relation $\sim$ described in (1). Then an $\mathrm{f}$-diophantine approximation is completely determined by the class ${ }^{*} f \in{ }^{*} \operatorname{Isom}\left(\mathbb{M}^{n}\right)$ of $\left\{f_{\alpha}\right\}$. We note that ${ }^{*} \operatorname{Isom}\left(\mathbb{M}^{n}\right)$ is a subgroup of $\operatorname{Isom}\left(\bullet \mathbb{M}^{n}\right)$ (the group of isometries of $\bullet \mathbb{M}^{n}$, not virtual isometries). Thus, if $\Gamma \cong \pi_{1} L$ is the deck group of $\mathbb{M}^{n} \rightarrow L$, we have

$$
{ }^{*} \Gamma \subset \llbracket \pi \rrbracket_{1}(\mathscr{L}, L, x, \mathrm{f}) \subset{ }^{*} \operatorname{Isom}\left(\mathbb{M}^{n}\right)
$$

The terminology f-diophantine approximation comes from the following example.

EXAMPLE 1. Let $\mathscr{L}$ be the irrational foliation of the torus $\mathbb{T}^{2}$ by lines of slope $r \in \mathbb{R} \backslash \mathbb{Q}$. Define a representation $\rho: \mathbb{Z} \cong \pi_{1} \mathbb{S}^{1} \rightarrow \operatorname{Homeo}\left(\mathbb{S}^{1}\right)$ by $\rho_{n}(\bar{y})=\overline{y-n r}$, where $\bar{y}$ denotes the image of $y \in \mathbb{R}$ in $\mathbb{S}^{1}=\mathbb{R} / \mathbb{Z}$. Then the suspension of $\rho, \mathscr{L}_{\rho}=\left(\mathbb{R} \times \mathbb{S}^{1}\right) / \mathbb{Z}$, is homeomorphic to $\mathscr{L}$. The 
$\operatorname{map} \mathbb{R} \times \mathbb{S}^{1} \rightarrow \mathbb{S}^{1}$ defined by $(x, \bar{y}) \mapsto \bar{x}$ (i.e. the projection onto the first factor composed with the universal covering $\mathbb{R} \rightarrow \mathbb{S}^{1}$ ) induces a projection $\mathscr{L}_{\rho} \rightarrow \mathbb{S}^{1}$. Let $T \approx \mathbb{S}^{1}$ be a fiber of this projection passing through $x$. A frame section $\mathrm{f}$ along $T$ is determined by an orientation of $\mathscr{L}$. In this case, an $\mathrm{f}$-diophantine approximation of $x$ is just a diophantine approximation of $r$. (Recall that a sequence $\left\{n_{\alpha}\right\} \subset \mathbb{Z}$ is called a diophantine approximation of $r \in \mathbb{R}$ if $\left\{\overline{r n_{\alpha}}\right\}$ converges to $\overline{0} \in \mathbb{S}^{1}$.) Thus if one denotes by ${ }^{*} \mathbb{Z}_{r}$ the subgroup of $* \mathbb{Z}$ consisting of classes of diophantine approximations of $r$, we obtain in agreement with the construction in $[4, \S 4.4]$ :

$$
\llbracket \pi \rrbracket_{1}(\mathscr{L}, L, x, \mathrm{f})={ }^{*} \mathbb{Z}_{r} .
$$

(Note: ${ }^{*} \mathbb{Z}_{r}$ is an ideal if and only if $r$ is rational.) If another frame field $\mathrm{f}^{\prime}$ is used whose domain is a transversal $T^{\prime}$ which is not a suspension fiber, the set of diophantine approximations is a subset ${ }^{*} \mathbb{R}_{r} \subset{ }^{*} \mathbb{R}$. This subset maps injectively into $\bullet \mathbb{R}$ with image $\bullet^{\mathbb{Z}_{r}}={ }^{*} \mathbb{Z}_{r}$.

Example 2. Consider a nested set of Fuchsian groups $\mathscr{G}=\left\{\Gamma_{i}\right\}$ and let

$$
\widehat{\Sigma}_{\mathscr{G}}=\lim _{\longleftarrow} \mathbb{H}^{2} / \Gamma_{i},
$$

be the associated hyperbolic surface solenoid. We may take $T$ to be a fiber $\hat{p}^{-1}\left(x_{0}\right)$ of the projection $\hat{p}: \widehat{\Sigma}_{\mathscr{G}} \rightarrow \Sigma_{0}$, where $\Sigma_{0}=\mathbb{H}^{2} / \Gamma_{0}$ is the initial surface. Then a frame at $x_{0}$ pulls back to a frame section $\mathrm{f}$ along $T$. In this case, we find that Definition 3 again agrees with the definition found in [4]:

$$
\begin{aligned}
& \llbracket \pi \rrbracket_{1}\left(\widehat{\Sigma}_{\mathscr{G}}, L, x, \mathrm{f}\right)=\bigcap^{*} \Gamma_{i} \\
& \quad=\left\{\left\{g_{\alpha}\right\} \subset \Gamma_{0} \mid \text { for all } i, \exists N_{i} \text { such that } g_{\alpha} \in \Gamma_{i} \text { when } \alpha>N_{i}\right\} / \sim,
\end{aligned}
$$

a subgroup of ${ }^{*} P S L(2, \mathbb{R}) \cong P S L\left(2,{ }^{*} \mathbb{R}\right)$. If $\mathrm{f}^{\prime}$ is another frame field, not necessarily with a fiber transversal domain, then the corresponding germ $\llbracket \pi \rrbracket_{1}\left(\widehat{\Sigma}_{\mathscr{G}}, L, x, \mathrm{f}^{\prime}\right)$ need not define a subgroup of $P S L\left(2,{ }^{*} \mathbb{R}\right)$ and particularly, need not be isomorphic to $\bigcap^{*} \Gamma_{i}$ (although the fundamental germs calculated with respect to $f$ and $f^{\prime}$ are in canonical bijection). The issue here is the non-uniform nature of the action of $\operatorname{PSL}(2, \mathbb{R})$ on $\mathbb{H}^{2}$. This problem will become moot through the replacement of the fundamental germ by the mother germ, Section 6 .

EXAMPLE 3. More generally, let $\mathscr{L}$ be any hyperbolic surface lamination. Then the fundamental germ $\llbracket \pi \rrbracket_{1}(\mathscr{L}, L, x, \mathrm{f})$ is a subset of $P S L\left(2,{ }^{*} \mathbb{R}\right)$. Equally, if $\mathscr{L}$ is a hyperbolic 3-lamination, $\llbracket \pi \rrbracket_{1}(\mathscr{L}, L, x, \mathrm{f}) \subset \operatorname{PSL}\left(2,{ }^{*} \mathbb{C}\right)$. 


\section{§4. The germ universal cover}

Let $L \subset \mathscr{L}$ be a fixed leaf. Denote by $p: \widetilde{L} \rightarrow L$ the universal cover. We recall the following definition [4]:

Definition 4. The germ universal cover of $\mathscr{L}$ along $L$ is the subspace $\llbracket \widetilde{\mathscr{L}} \rrbracket \subset \cdot \widetilde{L}$ defined by

$$
\llbracket \widetilde{\mathscr{L}} \rrbracket=\left\{\left\{\tilde{x}_{\alpha}\right\} \subset \widetilde{L} \mid\left\{p\left(\tilde{x}_{\alpha}\right)\right\} \text { converges in } \mathscr{L}\right\} / \simeq .
$$

We will denote elements of the germ universal cover by $\bullet \tilde{x}$. There is a natural projection

$$
\text { • } p: \llbracket \widetilde{\mathscr{L} \rrbracket} \longrightarrow \mathscr{L} \quad \cdot \tilde{x} \longmapsto \hat{x}=\lim p\left(\tilde{x}_{\alpha}\right)
$$

where $\left\{\tilde{x}_{\alpha}\right\}$ is a representative sequence in the class $\bullet \tilde{x}$. We will write $\lim \bullet \tilde{x}=\hat{x}$ if $\bullet p(\bullet \tilde{x})=\hat{x}$. Note that $\bullet p$ is surjective if and only if $L$ is dense and in general $\bullet^{\bullet} p$ maps onto the closure $\bar{L}$ of $L$, itself a sublamination of $\mathscr{L}$.

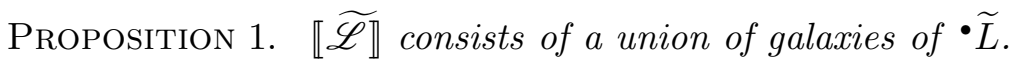

Proof. Let $\cdot \tilde{x} \in \llbracket \widetilde{\mathscr{L} \rrbracket}$ and denote by $U$ the galaxy containing $\bullet \tilde{x}$. If - $\tilde{y} \in U$, then there exists a sequence of geodesic paths $\left\{\tilde{\eta}_{\alpha}\right\}$ connecting representatives $\left\{\tilde{x}_{\alpha}\right\}$ to $\left\{\tilde{y}_{\alpha}\right\}$ in $\widetilde{L}$, whose projection to $\mathscr{L}$ gives a convergent sequence of paths $\left\{\eta_{\alpha}\right\}$. It follows that the projection $\left\{p\left(\tilde{y}_{\alpha}\right)\right\}$ converges, and $\bullet \tilde{y} \in \llbracket \widetilde{\mathscr{L}} \rrbracket$ as well.

The galaxies that make up $\llbracket \widetilde{\mathscr{L} \rrbracket}$ will be referred to as leaves. See Theorem 12 in [4] for a proof of the following

THEOREM 3. $\llbracket \widetilde{\mathscr{L} \rrbracket}$ may be given the structure of a lamination whose leaves are nowhere dense and such that the map ${ }^{\bullet} p: \llbracket \widetilde{\mathscr{L}} \rrbracket \rightarrow \bar{L}$ is an open surjection.

One can thus think of $\llbracket \widetilde{\mathscr{L} \rrbracket}$ as a the result of unwrapping all of the diophantine approximations implied by $L$. The topology that $\llbracket \widetilde{\mathscr{L}} \rrbracket$ obtains from its lamination atlas is not unique, and is called a germ universal cover topology. It is in general coarser than the topology $\llbracket \widetilde{\mathscr{L}} \rrbracket$ induces from $\cdot \widetilde{L}$.

Proposition 2. If $\mathscr{L}$ is compact then $\llbracket \widetilde{\mathscr{L} \rrbracket}=\cdot \widetilde{L}$. 
Proof. This follows from well-known compactness arguments e.g. see the proof of Proposition 14 in [4].

An element $\cdot u=\cdot f \circ g^{-1} \in \llbracket \pi \rrbracket_{1}(\mathscr{L}, L, x, \mathrm{f})$ arises as the limit of a sequence of $K_{\alpha}$-quasiisometries

$$
\left\{u_{\alpha}: D_{r_{\alpha}}\left(\tilde{x}_{\alpha}\right) \longrightarrow D_{r_{\alpha}}\left(\tilde{y}_{\alpha}\right)\right\}
$$

where $\left\{\tilde{x}_{\alpha}\right\},\left\{\tilde{y}_{\alpha}\right\} \subset L, K_{\alpha} \rightarrow 1$ and $r_{\alpha} \rightarrow \infty$. The limit $\bullet u: V \rightarrow U$ is independent of the sequence $\left\{u_{\alpha}\right\}$ and depends only on the sequences of frames $\left\{f_{x_{\alpha}}\right\},\left\{f_{y_{\alpha}}\right\}$. In particular, we could have obtained $\bullet u$ through the same sequence of quasiisometries with domains extended to a sequence of larger disks $D_{s_{\alpha}}\left(\tilde{x}_{\alpha}\right), s_{\alpha}>r_{\alpha}$ - provided that the new quasiisometry constants converge to 1 as well.

Now for arbitrary $\bullet \tilde{x} \in \llbracket \widetilde{\mathscr{L} \rrbracket}$, the expression $\bullet u(\bullet x)$ does not even make formal sense, since $\bullet u$ is so far only defined on the galaxy $V$. We contrast this with the constant curvature case, where, because $\llbracket \pi \rrbracket_{1}(\mathscr{L}, x, L, \mathrm{f}) \subset$ $\operatorname{Isom}(\bullet \mathbb{M}), \bullet u(\bullet \tilde{x})$ is always formally defined, although it need not define an element of $\llbracket \widetilde{\mathscr{L}} \rrbracket$.

Let us say that $\cdot u$ is formally defined on an element $\bullet \tilde{w} \in \llbracket \widetilde{\mathscr{L}} \rrbracket$ if there exists a sequence $(2)$ giving rise to $\bullet u$ and a representative sequence $\left\{\tilde{w}_{\alpha}\right\}$ of $\bullet \tilde{w}$ such that

$$
D_{r_{\alpha}^{\prime}}\left(w_{\alpha}\right) \subset D_{r_{\alpha}}\left(\tilde{x}_{\alpha}\right)
$$

for all $\alpha$, where $r_{\alpha}^{\prime} \rightarrow \infty$. It follows then that if $V^{\prime}$ is the galaxy containing $\bullet \tilde{w}$, then the limit $\bullet$ is defined on $V^{\prime}$ as well. Whenever we write $\bullet u(\bullet w)$, it will tacitly be understood that $\bullet u$ is formally defined at $\bullet$.

Define the domain of $\bullet u$ as

$$
\operatorname{Dom}(\bullet u)=\{\bullet \tilde{x} \in \llbracket \widetilde{\mathscr{L}} \rrbracket \mid \bullet u(\bullet \tilde{x}) \in \llbracket \widetilde{\mathscr{L}} \rrbracket \text { and } \lim \bullet u(\bullet \tilde{x})=\lim \bullet \tilde{x}\}
$$

and $\operatorname{Ran}(\bullet u)=\bullet u(\operatorname{Dom}(\bullet u))$. With this definition, it follows that $\llbracket \pi \rrbracket_{1}(\mathscr{L}, L, x, \mathrm{f})$ has the structure of a groupoid. Note that for any $\bullet \in$ $\llbracket \pi \rrbracket_{1}(\mathscr{L}, L, x, \mathrm{f}), \operatorname{Dom}(\bullet u), \operatorname{Ran}(\bullet u)$ are unions of leaves and hence induce lamination structures from $\llbracket \widetilde{\mathscr{L}} \rrbracket$. Moreover, on $\operatorname{Dom}(\bullet u)$,

$$
\bullet p \circ u=\bullet p .
$$

In particular we see that $\bullet u: \operatorname{Dom}(\bullet u) \rightarrow \operatorname{Ran}(\bullet u)$ defines a lamination homeomorphism. 
EXAMPLE 4 . Let $\mathscr{L}$ be the irrational foliation of $\mathbb{T}^{2}$ by lines of slope $r, L \approx \mathbb{R}$ any dense leaf. Then by Proposition $2, \llbracket \widetilde{\mathscr{L}} \rrbracket=\cdot \mathbb{R}$. Moreover, for any frame field $\mathrm{f}$ and $\bullet u \in \llbracket \pi \rrbracket_{1}(\mathscr{L}, L, x, \mathrm{f})$, it is not difficult to see that $\operatorname{Dom}(\bullet u)=\bullet \mathbb{R}$. Thus, $\llbracket \pi \rrbracket_{1}(\mathscr{L}, L, x, \mathrm{f})$ is a group isomorphic to $* \mathbb{Z}_{r}$.

EXAMPLE 5 . Let $\mathscr{L}$ be the profinite hyperbolic surface solenoid $\widehat{\Sigma}_{\mathscr{G}}$ of Example 2. Then we have, again by compactness,

$$
\llbracket \widetilde{\mathscr{L}} \rrbracket=\bullet \mathbb{H}^{2} .
$$

If $\mathrm{f}$ is a frame field lifted from a frame on a surface occurring in the defining inverse limit, then $\llbracket \pi \rrbracket_{1}(\mathscr{L}, L, x, \mathrm{f})$ is a group. On the other hand, if $\mathrm{f}$ is a frame field not obtained in this way, then $\llbracket \pi \rrbracket_{1}(\mathscr{L}, L, x, \mathrm{f})$ need not be a group e.g. see Section 5.

The proof of the following theorem is identical to that of Proposition 16 in [4].

TheOREM 4. Let $F:(\mathscr{L}, L) \rightarrow\left(\mathscr{L}^{\prime}, L^{\prime}\right)$ be a lamination map. Then there exist germ universal cover topologies so that the map

$$
\llbracket \widetilde{F} \rrbracket: \llbracket \widetilde{\mathscr{L}} \rrbracket \longrightarrow \llbracket \widetilde{\mathscr{L}}^{\prime} \rrbracket
$$

induced by $\left\{\tilde{x}_{\alpha}\right\} \mapsto\left\{\widetilde{F}\left(\tilde{x}_{\alpha}\right)\right\}$ is a continuous lamination map.

Note 2. It is useful here to point out that for a lamination $\mathscr{L}_{\rho}=(\widetilde{B} \times$ F) $/ \pi_{1} B$ occurring as a suspension of a representation $\rho: \pi_{1} B \rightarrow$ Homeo(F), it is in general false that a lamination homeomorphism $F: \mathscr{L}_{\rho} \rightarrow \mathscr{L}_{\rho}$ lifts to a homeomorphism of the "universal covering space" $\widetilde{B} \times \mathrm{F}$.

Now suppose that $L^{\prime}$ is another leaf of $\mathscr{L}$. Denote by $\llbracket \widetilde{\mathscr{L}} \rrbracket^{\prime}$ the germ universal cover formed from $L^{\prime}$.

Proposition 3. If $L^{\prime}$ accumulates on $L$ then there is a virtual subisometry $\bullet \widetilde{L} \rightarrow \cdot \widetilde{L}^{\prime}$ restricting to a virtual subisometry

$$
\llbracket \widetilde{\mathscr{L}} \rrbracket \longrightarrow \widetilde{\mathscr{L}} \rrbracket^{\prime}
$$

which is a homeomorphism onto its image with respect to appropriate germ universal cover topologies.

Proof. This follows directly from the proof of Theorem 2. 


\section{§5. Sensitivity to changes in data}

In this section we shall examine the dependence of the fundamental germ on the base point $x$, the accumulating leaf $L$ and the frame field $\mathrm{f}$.

Change in base point and accumulating leaf: Let us fix for the moment the dense leaf $L$ and consider a change of base point $x \mapsto x^{\prime}$ in which $L_{x}=L_{x^{\prime}}$. Let $\eta$ be a geodesic connecting $x$ to $x^{\prime}$ in $L_{x}$. The tangent vector $\vee$ to $\eta$ at $x$ has coordinate $\left(a_{1}, \ldots, a_{n}\right)$ with respect to the frame $\mathrm{f}_{x}$. At each $y \in T=$ the domain of $\mathrm{f}$, this coordinate determines a vector $\mathrm{v}_{y}$ using the frame $\mathrm{f}_{y}$. We obtain in this way a transversally continuous family of geodesics $\left\{\eta_{y}\right\}_{y \in T}$. Restricting to an open subtransversal of $T$ if necessary, we may parallel translate $f$ along the geodesic family to obtain a frame field $\mathrm{f}^{\prime}$ with domain $T^{\prime} \ni x^{\prime}$. The following is then immediate from the definition of the fundamental germ.

Proposition 4. Let $x^{\prime}, \mathrm{f}^{\prime}$ be as in the preceding paragraph. Then

$$
\llbracket \pi \rrbracket_{1}(\mathscr{L}, L, x, \mathrm{f})=\llbracket \pi \rrbracket_{1}\left(\mathscr{L}, L, x^{\prime}, \mathrm{f}^{\prime}\right) .
$$

If we consider a change of base point $x \mapsto x^{\prime}$, in which $L_{x} \neq L_{x^{\prime}}$, the situation becomes considerably more subtle. In fact, we shall see in Section 7 that fundamental germs based at points on different leaves can be nonisomorphic. For similar reasons, a change in accumulating leaf $L$ may yield nonisomorphic fundamental germs.

Change in frame field: Let us now fix the base point $x$ and consider a new frame field $\mathrm{f}^{\prime}: T^{\prime} \rightarrow \mathbf{F}_{*} \mathscr{L}$ based at $x$. For simplicity, we again assume that $\pi_{1} L=1$. Since $T$ (the domain of $\mathrm{f}$ ) and $T^{\prime}$ each contain subtransversal neighborhoods of $x$ lying in a common flow box, it is clear that there is a natural bijection

$$
\llbracket \pi \rrbracket_{1}(\mathscr{L}, L, x, \mathrm{f}) \longleftrightarrow \llbracket \pi \rrbracket_{1}\left(\mathscr{L}, L, x, \mathrm{f}^{\prime}\right)
$$

The issue is then the law of composition. We will show that this map need not be an isomorphism.

Let us consider the inverse limit solenoid $\widehat{\Sigma}_{\mathscr{G}}$ of Example 2. Assume that $L=L_{x}, T=T^{\prime}=$ a fiber over a point $x_{0} \in \Sigma_{0}$ and that $\mathrm{f}_{x}=\mathrm{f}_{x}^{\prime}$. We will take $\mathrm{f}$ to be simply the lift of a frame based at $x_{0}$, so that $\mathrm{f}$-diophantine approximations consist of sequences $\left\{\gamma_{\alpha}\right\} \subset \Gamma_{0}$ converging with respect to the family $\left\{\Gamma_{i}\right\}$. It follows that every $\mathrm{f}^{\prime}$-diophantine approximation of $x$ may be 
written in the form $\left\{\gamma_{\alpha} \Theta_{\alpha}\right\}$, where $\left\{\Theta_{\alpha}\right\}$ consists of a sequence of rotations based at $x$ with angle going to 0 and $\left\{\gamma_{\alpha}\right\}$ is an $\mathrm{f}$-diophantine approximation. General elements of $\llbracket \pi \rrbracket_{1}\left(\mathscr{L}, L, x, \mathrm{f}^{\prime}\right)$ are then of the form $\left\{\gamma_{\alpha} \Theta_{\alpha} \eta_{\alpha}\right\}$, where $\left\{\eta_{\alpha}\right\}$ is another $\mathrm{f}$-diophantine approximation. We should not expect products of elements of this type to yield elements of $\llbracket \pi \rrbracket_{1}\left(\mathscr{L}, L, x, \mathrm{f}^{\prime}\right)$. Indeed, such a product would have the shape

$$
\left\{\gamma_{\alpha} \Theta_{\alpha} \eta_{\alpha} \Delta_{\alpha} \omega_{\alpha}\right\}
$$

for $\left\{\Delta_{\alpha}\right\}$ another sequence of rotations based at $x$ with angle going to 0 and $\left\{\omega_{\alpha}\right\}$ another $\mathrm{f}$-diophantine approximation. If $\Theta_{\alpha}$ does not converge to the identity fast enough, $\Theta_{\alpha} \eta_{\alpha} \Delta_{\alpha} \omega_{\alpha}$ applied to $f_{x}^{\prime}$ will not project to a frame based at $x_{0} \in \Sigma_{0}$. Hence the expression (4) is not even asymptotic to an element of $\llbracket \pi \rrbracket_{1}\left(\mathscr{L}, L, x, \mathrm{f}^{\prime}\right)$. It is not difficult to see that unless $\mathrm{f}^{\prime}$ is the pull-back of a frame on $\Sigma_{0}$, this sort of problem always arises.

\section{§6. The mother germ}

In this section, we assume that $\mathscr{L}$ has a dense leaf $L$, with which we define the germ universal cover $\llbracket \widetilde{\mathscr{L}} \rrbracket$, equipped with a fixed germ universal cover topology.

While the fundamental germ $\llbracket \pi \rrbracket_{1}(\mathscr{L}, L, x, \mathrm{f})$ enjoys the property of being reasonably calculable and leaf specific, it can be sensitive to data variation. There are additional shortcomings:

- By $(3)$, the action of the fundamental germ $\llbracket \pi \rrbracket_{1}(\mathscr{L}, L, x, \mathrm{f})$ on $\llbracket \widetilde{\mathscr{L}} \rrbracket$ respects the germ covering $\bullet p$. However it need not be the case that every identification implied by $\bullet p$ is implemented by an element of $\llbracket \pi \rrbracket_{1}(\mathscr{L}, L, x, \mathrm{f})$.

- There will be in general other maps of leaves of $\llbracket \widetilde{\mathscr{L}} \rrbracket$ that satisfy $(3)$ but do not appear in $\llbracket \pi \rrbracket_{1}(\mathscr{L}, L, x, \mathrm{f})$.

- It appears that $\llbracket \pi \rrbracket_{1}(\mathscr{L}, L, x, \mathrm{f})$ such as it is defined, will be functorial only under certain types of lamination maps e.g. see Section 8 of [4].

For this reason, we will expand $\llbracket \pi \rrbracket_{1}(\mathscr{L}, L, x, \mathrm{f})$ to a larger groupoid, called the mother germ. The mother germ will be the maximal amplification of $\llbracket \pi \rrbracket_{1}(\mathscr{L}, L, x, \mathrm{f})$ which contains all partially defined maps of sublaminations of $\llbracket \widetilde{\mathscr{L}} \rrbracket$ satisfying $(3)$ : in other words, it is the full deck groupoid of $\bullet p$.

Let Dom, Ran be sublaminations of $\llbracket \widetilde{\mathscr{L}} \rrbracket$. A homeomorphism

$$
\text { • } u: \text { Dom } \longrightarrow \operatorname{Ran} \subset \llbracket \widetilde{\mathscr{L}} \rrbracket
$$


satisfying (3) is called deck. Note that condition (3) implies that a deck homeomorphism $\bullet u$ is automatically an isometry along the leaves of Dom.

Definition 5. The mother germ is the groupoid

$$
\llbracket \pi \rrbracket_{1}(\mathscr{L})=\{\bullet u: \text { Dom } \rightarrow \text { Ran is a deck homeomorphism }\}
$$

The mother germ will never be a group, since it distinguishes deck maps obtained from others by restriction of domain. In general, however, it will contain many interesting and calculable subgroups and subgroupoids, as the following shows.

Proposition 5. Let $L^{\prime}$ be any dense leaf of $\mathscr{L}$. Then there is an injective groupoid homomorphism

$$
\llbracket \pi \rrbracket_{1}\left(\mathscr{L}, L^{\prime}, x, \mathrm{f}\right) \longleftrightarrow \llbracket \pi \rrbracket_{1}(\mathscr{L}) .
$$

Proof. By Proposition 3, there exists an isometric inclusion $\bullet f$ : $\llbracket \widetilde{\mathscr{L}} \rrbracket^{\prime} \hookrightarrow \llbracket \widetilde{\mathscr{L}} \rrbracket$. If $\bullet u \in \llbracket \pi \rrbracket_{1}\left(\mathscr{L}, L^{\prime}, x, \mathrm{f}\right)$, then the map

$$
\bullet u \longmapsto \cdot f \circ \cdot u \circ \cdot f^{-1}
$$

defines an injective groupoid homomorphism.

THEOREM 5. The quotient

$$
\llbracket \pi \rrbracket_{1}(\mathscr{L}) \backslash \llbracket \widetilde{\mathscr{L} \rrbracket}
$$

equipped with the quotient germ universal cover topology, has the structure of a riemannian lamination canonically isometric to $\mathscr{L}$.

Proof. Let $\bullet \tilde{x}, \bullet \tilde{y} \in \llbracket \widetilde{\mathscr{L} \rrbracket}$ be $\operatorname{such}$ that $\lim \bullet \tilde{x}=\lim \bullet \tilde{y}=x$. Thus each point is represented by sequences in $\widetilde{L}$ that project to sequences $\left\{x_{\alpha}\right\}$, $\left\{y_{\alpha}\right\} \subset L$ having a common limit $x$. Let $\mathrm{f}$ be a frame field along a transversal $T$ containing $x$ and which we may assume contains $\left\{x_{\alpha}\right\}$ and $\left\{y_{\alpha}\right\}$. Then if $\bullet f, \bullet g$ are the diophantine approximations associated to $\left\{x_{\alpha}\right\},\left\{y_{\alpha}\right\}$ we have $\bullet=\bullet^{\bullet} g \bullet^{-1} \in \llbracket \pi \rrbracket_{1}(\mathscr{L}, L, x, f)$ identifies $\bullet \tilde{x}$ with $\bullet \tilde{y}$. Since this latter groupoid belongs to the mother germ by Proposition 5 , it follows that $\llbracket \pi \rrbracket_{1}(\mathscr{L}) \backslash \llbracket \widetilde{\mathscr{L} \rrbracket}$ contains all of the identifications implied by $\bullet^{*} p$ and so may be identified with $\mathscr{L}$ with its quotient topology. 
Let $\mathscr{L}, \mathscr{L}^{\prime}$ be riemannian laminations with dense leaves $L, L^{\prime}$. A map $\llbracket \widetilde{F} \rrbracket: \llbracket \widetilde{\mathscr{L}} \rrbracket \rightarrow \llbracket \widetilde{\mathscr{L}}^{\prime} \rrbracket$ is called standard if it is induced by $F: \widetilde{L} \rightarrow \widetilde{L}^{\prime}$ (e.g. compare with the definition found in Section 2). In addition, $\llbracket \widetilde{F} \rrbracket$ is called $\llbracket \pi \rrbracket_{1}(\mathscr{L})$-equivariant if there exists a groupoid homomorphism $\llbracket F \rrbracket_{*}$ : $\llbracket \pi \rrbracket_{1}(\mathscr{L}) \rightarrow \llbracket \pi \rrbracket_{1}\left(\mathscr{L}^{\prime}\right)$ such that

$$
\llbracket \widetilde{F} \rrbracket(\bullet u \cdot \bullet \tilde{x})=\llbracket F \rrbracket *(\bullet u) \cdot \llbracket \widetilde{F} \rrbracket(\bullet \tilde{x})
$$

for all $\bullet u \in \llbracket \pi \rrbracket_{1}(\mathscr{L})$ and $\bullet \tilde{x} \in \operatorname{Dom}(\bullet u)$.

TheOrem 6. Let $\llbracket \widetilde{F} \rrbracket: \llbracket \widetilde{\mathscr{L}} \rrbracket \rightarrow \llbracket \widetilde{\mathscr{L}^{\prime} \rrbracket}$ be a standard, $\llbracket \pi \rrbracket_{1}(\mathscr{L})$-equivariant map. Then $\llbracket \widetilde{F} \rrbracket$ covers a unique map $F: \mathscr{L} \rightarrow \mathscr{L}^{\prime}$.

Proof. By equivariance, the expression

$$
F=\cdot p^{\prime} \circ \llbracket \widetilde{F} \rrbracket \circ p^{-1}
$$

yields a well-defined function $F: \mathscr{L} \rightarrow \mathscr{L}^{\prime}$, continuous because ${ }^{\bullet} p,{ }^{\bullet} p^{\prime}$ are open maps and $\llbracket \widetilde{F} \rrbracket$ is continuous.

In [4], functoriality of the fundamental germ was demonstrated only with respect to the restricted class of trained lamination maps. The following theorem shows that the mother germ is considerably more flexable. A lamination covering map is a surjective lamination map which is a covering map when restricted to any leaf.

TheOREM 7. Let $F: \mathscr{L} \rightarrow \mathscr{L}^{\prime}$ be a lamination covering map. Then $F$ induces an injective homomorphism of mother germs

$$
\llbracket F \rrbracket_{*}: \llbracket \pi \rrbracket_{1}(\mathscr{L}) \longleftrightarrow \llbracket \pi \rrbracket_{1}\left(\mathscr{L}^{\prime}\right) .
$$

Proof. Let $L \subset \mathscr{L}$ be a dense leaf and let $\widetilde{F}: \widetilde{L} \rightarrow \widetilde{L}^{\prime}$ be the leaf universal cover lift. Then by Theorem $4, \widetilde{F}$ induces a standard map

$$
\llbracket \widetilde{F} \rrbracket: \llbracket \widetilde{\mathscr{L}} \rrbracket \longrightarrow \llbracket \widetilde{\mathscr{L}}^{\prime} \rrbracket .
$$

We note that since $\widetilde{F}$ is injective, $\llbracket \widetilde{F} \rrbracket$ is a homeomorphism onto its image lamination. Let $\bullet u \in \llbracket \pi \rrbracket_{1}(\mathscr{L})$. Then the map

$$
\llbracket F \rrbracket_{*}(\bullet):=\llbracket \widetilde{F} \rrbracket \circ \bullet u \circ \llbracket \widetilde{F} \rrbracket^{-1},
$$


defined on $\llbracket \widetilde{F} \rrbracket(\operatorname{Dom}(\bullet u))$, is deck for the germ universal covering $\bullet p^{\prime}$. Indeed

$$
\begin{aligned}
\cdot p^{\prime} \circ\left(\llbracket \widetilde{F} \rrbracket \circ \bullet u \circ \llbracket \widetilde{F} \rrbracket^{-1}\right) & =F \circ(\bullet p \circ \cdot u) \circ \llbracket \widetilde{F} \rrbracket^{-1} \\
& =F \circ p \circ \llbracket \widetilde{F} \rrbracket^{-1} \\
& =\cdot p^{\prime} .
\end{aligned}
$$

Thus the map $\llbracket F \rrbracket_{*}$ is an injective groupoid homomorphism, and we are done.

We have the following

COROLlary 2. The mother germ $\llbracket \pi \rrbracket_{1}(\mathscr{L})$ is independent of leaf-wise riemannian metric and smooth structure. In particular, $\llbracket \pi \rrbracket_{1}(\mathscr{L})$ is a topological invariant.

\section{§7. The antenna lamination}

In this section, we will calculate the fundamental germ of the antenna Riemann surface lamination of Kenyon and Ghys [6]: it is distinguished by the unusual property of having dense leaves of both planar and hyperbolic conformal type.

We begin by constructing a graphical model of a dense leaf of the antenna lamination. Let $T_{1}$ be the cross with vertices $V_{1}=\{(0,0),( \pm 1,0)$, $(0, \pm 2)\}$ and edges consisting of the line segments connecting $(0,0)$ to each of the other four vertices. Suppose that we have constructed $T_{n}$ meeting the $x$-axis in the interval $\left[-2^{n}+1,2^{n}-1\right] \times\{0\}$ and meeting the $y$-axis in the interval $\{0\} \times\left[-2^{n}, 2^{n}\right]$. Translate $T_{n}$ vertically so that the origin is taken to $\left(0,2^{n}\right)$ and consider the images of this translate by rotations of the plane - about the origin - of angles $0, \pm \pi / 2, \pi$. The union of these images forms a tree; $T_{n+1}$ is then obtained by replacing the extremal edges $\left[2^{n+1}-2,2^{n+1}\right] \times\{0\}$ and $\left[-2^{n+1},-2^{n+1}+2\right] \times\{0\}$ by $\left[2^{n+1}-2,2^{n+1}-1\right] \times\{0\}$ and $\left[-2^{n+1}+1,-2^{n+1}+2\right] \times\{0\}$. It follows that $T_{1} \subset T_{2} \subset \cdots$ : we then define

$$
T_{\infty}=\lim _{\longrightarrow} T_{n}
$$

See Figure 1

Given $n \in \mathbb{Z}$, let $\operatorname{ord}_{2}(n)$ be the 2-adic order: the largest nonnegative integer $r$ for which $2^{r}$ divides $n$. Then the vertex set of $T_{\infty}$ is

$$
V_{\infty}=\{(0,0)\} \cup\left\{v=(x, y) \in \mathbb{Z} \oplus \mathbb{Z} \mid \operatorname{ord}_{2}(x) \neq \operatorname{ord}_{2}(y)\right\}
$$




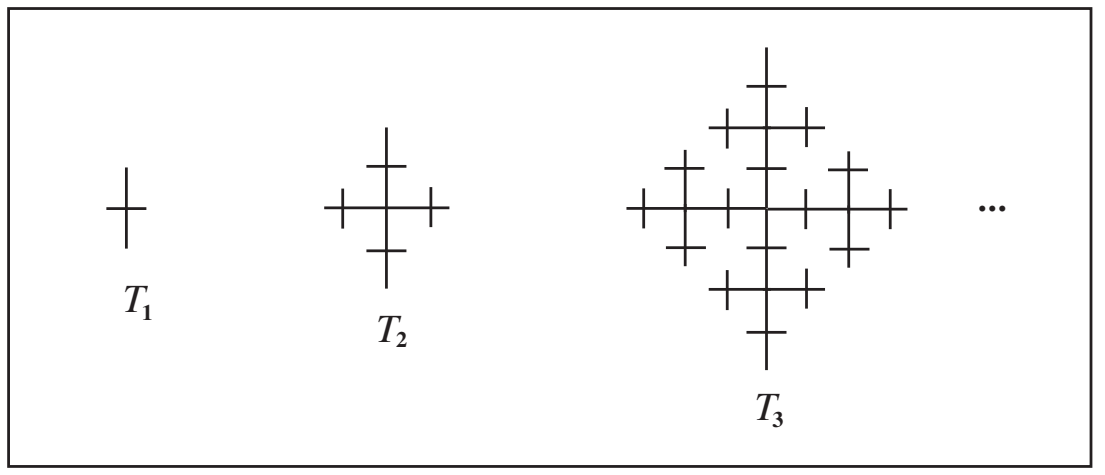

Figure 1: The Antenna Tree

We may view $V_{\infty}$ as a groupoid through its action on itself by addition. In order to avoid confusion, we write $v \circ w$ to indicate groupoid composition, in order to distinguish it from the element $v+w \in \mathbb{Z} \oplus \mathbb{Z}$.

Proposition 6. For all $v, w \in V_{\infty}$, the composition $v \circ w$ is defined if and only if $v=-w$.

Proof. Let $v, w \in V_{\infty}$. We show that $\operatorname{Ran}(w)=\operatorname{Dom}(v)$ if and only if $v=-w$. Suppose $v \neq-w$. Then we may write

$$
v+w=\left(\sum_{\alpha=m}^{M} a_{\alpha} 2^{\alpha}, \sum_{\alpha=n}^{N} b_{\alpha} 2^{\alpha}\right)
$$

where $m, n$ are the first non-zero indices of the 2-adic expansions of the coordinates. If $v \circ w$ is defined, then since $0 \in \operatorname{Dom}(w)$, we must have $v+w \in V_{\infty}$. In particular, at least one of $m$ or $n$ is nonzero. Suppose it is $n$; we may assume without loss of generality that $m<n$. Write

$$
w=\left(\sum_{\alpha=r}^{R} c_{\alpha} 2^{\alpha}, \sum_{\alpha=s}^{S} d_{\alpha} 2^{\alpha}\right) .
$$

Let $x=\left(0,2^{m}\right)$. If $r<m$, then $x \in \operatorname{Dom}(w)$ but $v+w+x \notin V_{\infty}$ i.e. $\operatorname{Ran}(w) \neq \operatorname{Dom}(v)$. This is also true when $r \geq m$ except for two cases. If $r>m$ and $s=m, w+x$ is not defined presisely when $1=d_{s}=\cdots=d_{r-1}$ and $d_{r}=0$. Here we take $x^{\prime}=\left(2^{r}, 2^{m}\right) \in \operatorname{Dom}(w)$ and note that $v+w+x^{\prime} \notin$ 
$V_{\infty}$. If $r=m$ and $s>m$, then $w+x$ is not defined. In this case, it follows from the form of $v+w$ that if $v=\left(v_{1}, v_{2}\right)$ then $\operatorname{ord}_{2}\left(v_{1}\right)>m$, so that $x \in \operatorname{Dom}(v)$. On the other hand, $x-w \notin \operatorname{Dom}(w)$. Thus $\operatorname{Ran}(w) \neq \operatorname{Dom}(v)$ here as well.

The lines $x= \pm y$ intersect $T_{\infty}$ at the origin only. Each of the four components of $T_{\infty} \backslash\{(0,0)\}$ defines an end, one contained in each of the four components of $\mathbb{R}^{2} \backslash\{(x, \pm x) \mid x \in \mathbb{R}\}$. Equipped with the path metric induced from $\mathbb{R}^{2}, T_{\infty}$ has exactly four orientation preserving isometries, corresponding to the rotations about the origin of angles $0, \pm \pi / 2, \pi$ (since ends must be taken to ends). On the other hand, $T_{\infty}$ has many partially defined isometries. For example, for $v \in V_{\infty}$, let $I_{v}$ be the map of $\mathbb{Z} \oplus \mathbb{Z}$ defined

$$
I_{v}(x, y)=v+(x, y) .
$$

Then there is a maximal subtree $T_{\infty}^{v} \subset T_{\infty}$ (not necessarily connected) for which $I_{v}\left(T_{\infty}^{v}\right) \subset T_{\infty}$. By definition, $I_{v}$ is isometric on its domain of definition. If $v$ has coordinates of large 2-adic order, then $I_{v}$ is defined on a large ball about 0 in $T_{\infty}$. More precisely, if $v=(x, y)$ and $\operatorname{ord}_{2}(x), \operatorname{ord}_{2}(y) \geq$ $n$ then $T_{n} \subset T_{\infty}^{v}$. Although the inverse $I_{v}^{-1}=I_{-v}$ is always defined at 0 , the composition $I_{v_{1}} \circ I_{v_{2}}=I_{v_{1}+v_{2}}$ will not be defined at 0 if $v_{1}+v_{2} \notin V_{\infty}$.

We now define a riemannian surface modelled on $T_{\infty}$, which will occur as a dense leaf of the antenna lamination. Regarding $T_{\infty} \subset \mathbb{R}^{2} \times\{0\} \subset \mathbb{R}^{3}$, it is clear that

$$
S_{\infty}=\text { boundary of a tubular neighborhood of } T_{\infty}
$$

is homeomorphic to a sphere with four punctures. We want to fix a particular realization of $S_{\infty}$ so that the partial isometries $I_{v}$ of $T_{\infty}$ will induce partial isometries of $S_{\infty}$. Torward this end, consider the surfaces shown in Figure 2.

We assume that they are equipped with riemannian metrics and boundary parametrizations so that given any pair of such surfaces and a choice of boundary component of each, the glueings are canonical and isometric. Each riemannian surface corresponds to a subgraph of $T_{\infty}$, and we may build $S_{\infty}$ from these riemannian surfaces using $T_{\infty}$ as a template. The metrics on the building blocks will also be chosen so that when $S_{\infty}$ is assembled within $\mathbb{R}^{3}$ it is invariant not only with respect to $\pi / 2$-rotations about the $z$-axis, but also $\pi$-rotations about the $x$ and $y$-axes. We think of $T_{\infty}$ as 


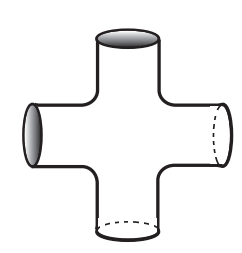

4 holed sphere

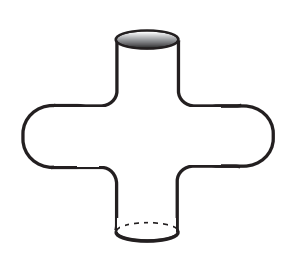

cylinder

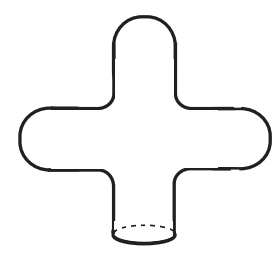

disk

Figure 2: Building Blocks

a spine floating inside the tubular neighborhood bounded by $S_{\infty}$, and we project in the positive vertical direction a copy of $T_{\infty}$ onto $S_{\infty}$. We denote this copy also by $T_{\infty}$, and use the symbols 0 and $v$ to denote the origin and a generic element of its vertex set as well. Having constructed $S_{\infty}$ in this way, it is clear that every $I_{v}$ induces a partial isometry of $S_{\infty}$ whose domain is the subsurface (with boundary) of $S_{\infty}$ modelled on $T_{\infty}^{v}$. We denote this partial isometry $I_{v}$ as well.

Let $S_{\infty}^{+}$be the intersection of $S_{\infty}$ with the half plane $z \geq 0$. The universal cover $\widetilde{S}_{\infty}$ of $S_{\infty}$ is built up from "tiles" modelled on $S_{\infty}^{+}$, glued together side by side according to the same pattern one uses to glue ideal quadrilaterals to obtain the hyperbolic plane as the universal cover of the four times punctured sphere. Fix $\tilde{0} \in \widetilde{S}_{\infty}$ a base point lying over 0 . The deck group of the universal covering map is $F_{3}$, the free group on three generators.

Let $\mathrm{w}_{0}$ be the unit vector based at 0 which is parallel to the $x$-axis and points in the positive direction. Consider the vector field $W$ on the vertices of $T_{\infty}$ obtained by parallel translating $\mathrm{w}_{0}$ along $T_{\infty}$. Note that the partial isometry of $S_{\infty}$ induced by $I_{v}, v \in V_{\infty}$, takes $\mathrm{w}_{0}$ to $\mathrm{w}_{v}=\mathrm{W}(v)$. This is not true of the rotations by angles $\pm \pi / 2$ and $\pi$.

Let $\mathrm{D}_{\tilde{0}} \subset \widetilde{S}_{\infty}$ be the fundamental domain containing $\tilde{0}$. We lift $T_{\infty}$ to $\mathrm{D}_{\tilde{0}}$, then translate it by $F_{3}$ to obtain a (disconnected) graph $\widetilde{T}_{\infty}$ on $\widetilde{S}_{\infty}$. Let $\widetilde{W}$ be the vector field defined on the vertices of $\widetilde{T}_{\infty}$ that is the lift of W. The partial isometry $I_{v}$ lifts to a partial isometry of $\widetilde{S}_{\infty}$ which maps a region of each fundamental domain D into D: we denote this privileged lift by $I_{v}$ 
as well, and the set of such privileged lifts is denoted $\mathbb{I}$. In addition, by composing with elements of $F_{3}$, we obtain new partial isometries covering $I_{v}: S_{\infty} \rightarrow S_{\infty}$. We denote by $\widetilde{\mathbb{I}}$ the set of partial isometries of $\widetilde{S}_{\infty}$ obtained in this way. Then $F_{3}, \mathbb{I} \subset \widetilde{\mathbb{I}}$, and every element of $F_{3}$ commutes with every element of $\mathbb{I}$.

We are now ready to describe the antenna lamination. Consider first the space $\mathbb{A}$ of all trees in $\mathbb{R}^{2}$ whose vertex set contains the origin 0 and lies within $\mathbb{Z} \oplus \mathbb{Z}$. Each tree $T \in \mathbb{A}$ is equipped with the path metric induced from $\mathbb{R}^{2}$. On $\mathbb{A}$, we consider the metric

$$
d\left(T, T^{\prime}\right)=\exp (-n)
$$

where $n$ is the largest integer such that the ball of radius $n$ about 0 in $T$ coincides with that about 0 in $T^{\prime}$. $\mathbb{A}$ is a compact metric space, [6]. Two graphs $T$ and $T^{\prime}$ are termed equivalent if there exists a translation by $(x, y) \in \mathbb{Z} \oplus \mathbb{Z}$ such that $T+(x, y)=T^{\prime}$.

Now for any tree $T \in \mathbb{A}$, the ball of radius 1 about 0 is a tree $P \in \mathbb{A}$ all of whose vertices lie in the set $\{(0,0)\} \cup\{( \pm 1, \pm 1)\}$. We write $|P| \leq 4$ for the number of vertices $v$ of $P$ different from 0 . There are 16 possible such $P$, and we may decompose $\mathbb{A}$ into a disjoint union of clopens $\mathbb{A}_{P}$, where $\mathbb{A}_{P}$ consists of those trees whose unit ball about 0 is $P$.

For each $P$, we consider in the spirit of Figure 2 a model pointed Riemann surface $\left(\Sigma_{P}, z_{P}\right)$ homeomorphic to $\mathbb{S}^{2} \backslash(|P|$ open disks $)$. We assume as before that each boundary component $\partial_{v} \Sigma_{P}$ - labeled by a vertex $v \neq(0,0)$ of $P$ - has a fixed parametrization, so that any two may be identified along their boundaries without ambiguity. Define

$$
\mathscr{L}=\left(\bigcup\left(\mathbb{A}_{P} \times \Sigma_{P}\right)\right) / \text { gluing, }
$$

where the gluing is performed as follows. Given $T \in \mathbb{A}_{P}, v \in P$, the translate $T+v$ is in $\mathbb{A}_{P^{\prime}}$ for some $P^{\prime}$, where $-v \in P^{\prime}$. We then glue the boundaries $\partial_{v} \Sigma_{P}$ and $\partial_{-v} \Sigma_{P^{\prime}}$. These gluings are compatible with the trivial lamination structures on the $\mathbb{A}_{P} \times \Sigma_{P}$ and thus $\mathscr{L}$ has the structure of a riemannian surface lamination. Note that there is an embedding $\mathbb{A} \hookrightarrow \mathscr{L}$ induced by $\mathbb{A}_{P} \times\left\{z_{P}\right\} \hookrightarrow \mathbb{A}_{P} \times \Sigma_{P}$.

Each leaf $L \subset \mathscr{L}$ corresponds to an equivalence class of graph $T \in \mathbb{A}$, embedded in $L$ as a spine. Note that $S_{\infty}$ is the leaf corresponding to the class of $T_{\infty}$. Define the antenna lamination $\mathscr{L}_{\infty}$ to be the closure of $S_{\infty}$ in $\mathscr{L}$. 
Denote by $S_{n} \subset S_{\infty}$ the surface (with boundary) modelled on the subgraph $T_{n} \subset T_{\infty}$. If centered at a vertex $v \in V_{\infty}$ there is a subgraph isometric to $T_{n}$, it models a subsurface $S_{n}(v)$ containing $v$, and the isometry $I_{v}$ maps $S_{n}$ to $S_{n}(v)$.

The closure of $V_{\infty}$ in $\mathscr{L}_{\infty}$ defines a transversal $\mathrm{T}$ through $0 \in S_{\infty}$, and the vector field $W$ is transversally continuous with respect to the topology of T. A point $v \in V_{\infty}$ is transversally close to 0 if and only if its coordinates have large 2-adic order.

We are now ready to calculate the fundamental germ

$$
\llbracket \pi \rrbracket_{1}\left(\mathscr{L}_{\infty}, 0, \mathrm{f}\right)
$$

where $f$ is the orthonormal frame field determined by $W$.

Define nested sets

$$
\widetilde{G}=\left\{\widetilde{G}_{n}\right\} \subset \widetilde{\mathbb{I}} \text { and } G=\left\{G_{n}\right\} \subset \mathbb{I},
$$

$n=0,1,2, \ldots$, as follows. We say that $\widetilde{I} \in \widetilde{\mathbb{I}}$ is $n$-close if the domain of $\widetilde{I}$ contains the finite tree $\widetilde{T}_{n} \subset \widetilde{T}_{\infty} \cap \mathrm{D}_{\tilde{0}}$ corresponding to $T_{n}$, and maps it into the fundamental domain containing $\widetilde{I}(\tilde{0})$. Then $\widetilde{G}_{n}$ consists of the set of $n$-close maps and $G_{n}$ the $n$-close maps in $\mathbb{I}$. Observe that

$$
F_{3}=\bigcap \widetilde{G}_{n}
$$

For $I \in G_{n}, I^{-1} \in G_{n}$ also. Moreover, if $I^{\prime} \in G_{m}$ and the composition $I \circ I^{\prime}$ is defined at $\tilde{0}$, then it belongs to $G_{N}$, for $N=\min (m, n)$.

Let

$$
\begin{aligned}
\llbracket G \rrbracket=\left\{\left\{I_{v_{\alpha}} \circ I_{v_{\alpha}^{\prime}}^{-1}\right\}=\left\{I_{v_{\alpha}-v_{\alpha}^{\prime}}\right\} \mid\right. \\
\left.\left\{I_{v_{\alpha}}\right\},\left\{I_{v_{\alpha}^{\prime}}\right\} \subset \mathbb{I} \text { and converge w.r.t. } G\right\} / \sim,
\end{aligned}
$$

where the relation $\sim$ is defined by an ultrafilter $\mathfrak{U}$ as in $(1)$. We denote the elements of $\llbracket G \rrbracket$ by $I^{*} v$ where ${ }^{*} v \in{ }^{*} \mathbb{Z} \oplus{ }^{*} \mathbb{Z}$, and regard $\llbracket G \rrbracket$ as a groupoid with unit space $\llbracket \widetilde{L}_{\infty} \rrbracket$, in which the domains of elements are taken to be maximal in the sense defined in Section 4.

Proposition 7. As a set, $\llbracket G \rrbracket$ may be identified with ${ }^{*} \mathbb{Z}_{\hat{2}} \oplus^{*} \mathbb{Z}_{\hat{2}}$, where

$$
* \mathbb{Z}_{\hat{2}}=\left\{\left\{n_{\alpha}\right\} \subset \mathbb{Z} \mid \operatorname{ord}_{2}\left(n_{\alpha}\right) \rightarrow \infty \text { as } \alpha \rightarrow \infty\right\} / \sim \text {. }
$$

Given ${ }^{*} v,{ }^{*} w \in{ }^{*} \mathbb{Z}_{\hat{2}} \oplus^{*} \mathbb{Z}_{\hat{2}}$, the composition $I^{*} v \circ I^{*} w$ is defined if and only if ${ }^{*} v=-{ }^{*} w$. 
Proof. Any element $\left({ }^{*} n_{1},{ }^{*} n_{2}\right) \in{ }^{*} \mathbb{Z}_{\hat{2}} \oplus{ }^{*} \mathbb{Z}_{\hat{2}}$ may be written $\left({ }^{*} n_{1}, 0\right)-$ $\left(0,-{ }^{*} n_{2}\right)$ which clearly defines an element of $\llbracket G \rrbracket$. Now consider ${ }^{*} v,{ }^{*} w \in$ ${ }^{*} \mathbb{Z}_{\hat{2}} \oplus{ }^{*} \mathbb{Z}_{\hat{2}}$, and suppose that ${ }^{*} v \neq-{ }^{*} w$. The 2 -adic order extends to

$$
\operatorname{ord}_{2}:{ }^{*} \mathbb{Z}_{\hat{2}} \longrightarrow{ }^{*} \mathbb{Z}_{\infty}=\left({ }^{*} \mathbb{Z} \backslash \mathbb{Z}\right) \cup\{0\}
$$

Let ${ }^{*} V_{\infty} \subset{ }^{*} \mathbb{Z}_{\hat{2}} \oplus{ }^{*} \mathbb{Z}_{\hat{2}}$ be the subset of pairs ${ }^{*} u=\left({ }^{*} u_{1},{ }^{*} u_{2}\right)$ for which $\operatorname{ord}_{2}\left({ }^{*} u_{1}\right) \neq \operatorname{ord}_{2}\left({ }^{*} u_{2}\right)$. We distinguish four cases depending on whether ${ }^{*} v,{ }^{*} w \in{ }^{*} V_{\infty}$ or not. If ${ }^{*} v,{ }^{*} w \in{ }^{*} V_{\infty}$ then we may regard each as a class of sequences $\left\{v_{\alpha}\right\},\left\{w_{\alpha}\right\} \subset V_{\infty}$. If $\left\{m_{\alpha}\right\},\left\{n_{\alpha}\right\},\left\{r_{\alpha}\right\},\left\{s_{\alpha}\right\}$ are the sequences of indices occurring as in Proposition 6 , then their classes ${ }^{*} m,{ }^{*} n,{ }^{*} r,{ }^{*} s$ are totally ordered in $* \mathbb{Z}$, hence we may assume the representative sequences are. In particular, we may proceed with the same argument as in Proposition 6: the sequences $\left\{x_{\alpha}\right\},\left\{x_{\alpha}^{\prime}\right\}$ define elements of $\llbracket \mathscr{L}_{\infty} \rrbracket$ which may be used to show that the composition $I_{*} v \circ I_{*} w$ is not defined. Now suppose that ${ }^{*} v \notin$ ${ }^{*} V_{\infty}$ but ${ }^{*} w \in{ }^{*} V_{\infty}$. This means that both components of ${ }^{*} v$ have the same order denoted $\operatorname{ord}_{2}\left({ }^{*} v\right)$. Then there exists ${ }^{*} x \in{ }^{*} V_{\infty}$ such that ${ }^{*} w+{ }^{*} x \in{ }^{*} V_{\infty}$ in which the two components of ${ }^{*} w+{ }^{*} x$ have order greater than $\operatorname{ord}_{2}\left({ }^{*} v\right)$. Then both components of ${ }^{*} v+{ }^{*} w+{ }^{*} x$ have equal order, which implies that $I^{*} v \circ I^{*} w$ is not defined. The case where ${ }^{*} v \in{ }^{*} V_{\infty}$ but ${ }^{*} w \notin{ }^{*} V_{\infty}$ is handled similarly. Now suppose ${ }^{*} v,{ }^{*} w \notin{ }^{*} V_{\infty}$. Here there are two subcases. First suppose that the orders of the components of ${ }^{*} v,{ }^{*} w$ are not equal. Denote by $\operatorname{ord}_{2}\left({ }^{*} v\right), \operatorname{ord}_{2}\left({ }^{*} w\right)$ the common order of the components of ${ }^{*} v$, ${ }^{*} w$. Then if say $\operatorname{ord}_{2}\left({ }^{*} v\right)<\operatorname{ord}_{2}\left({ }^{*} w\right)$, we define ${ }^{*} x=\left(0,{ }^{*} w_{2}\right)$ where ${ }^{*} w_{2}$ is the second component of ${ }^{*} w$. Then $I_{*} w\left({ }^{*} x\right)$ is defined but $I^{*} v+{ }^{*} w\left({ }^{*} x\right)$ is not. If $\operatorname{ord}_{2}\left({ }^{*} v\right)>\operatorname{ord}_{2}\left({ }^{*} w\right)$ then $I_{*} v$ is defined on ${ }^{*} y=\left(0,{ }^{*} v_{2}\right)$ but ${ }^{*} y-{ }^{*} w$ does not define an element of $\operatorname{Dom}\left(I_{*} w\right)$ since it does not converge to the same point in $\llbracket \widetilde{\mathscr{L}}_{\infty} \rrbracket$ as ${ }^{*} y$. What remains is the case when $\operatorname{ord}_{2}\left({ }^{*} v\right)=\operatorname{ord}_{2}\left({ }^{*} w\right)$. If ${ }^{*} v+{ }^{*} w$ lies in ${ }^{*} V_{\infty}$ then ${ }^{*} w \in \operatorname{Dom}\left(I_{*}\right)$ but not in $\operatorname{Ran}\left(I^{*} w\right)$. Otherwise, if the norms of the components of ${ }^{*} v+{ }^{*} w$ are equal, then $\operatorname{ord}_{2}\left({ }^{*} v+{ }^{*} w\right)>$ $\operatorname{ord}_{2}\left({ }^{*} v\right)=\operatorname{ord}_{2}\left({ }^{*} w\right)$. If we let ${ }^{*} x=\left(\left({ }^{*} v+{ }^{*} w\right)_{1}, 0\right)$ then ${ }^{*} x \in \operatorname{Dom}\left(I^{*} v+{ }^{*} w\right)$ but not in $\operatorname{Dom}\left(I^{*} w\right)$ so it cannot be that $I_{*} v \circ I^{*} w=I{ }^{*} v{ }^{*} w$.

THEOREM 8. As a set

$$
\llbracket \pi \rrbracket_{1}\left(\mathscr{L}_{\infty}, 0, \mathrm{f}\right)={ }^{*} F_{3} \times \llbracket G \rrbracket .
$$

The composition $\bullet \cdot \bullet^{\bullet} v$, where $\cdot v=\left({ }^{*} x, I^{*} v\right), \cdot w=\left({ }^{*} y, I^{*} w\right)$ is defined if and only if ${ }^{*} v=-{ }^{*} w$. 
Proof. Every element $\widetilde{\mathbb{I}}$ may be written in the form $I_{v} \circ \gamma=\gamma \circ I_{v}$ for $v \in V_{\infty}$ and $\gamma \in F_{3}$. Moreover, if $\widetilde{I} \in \widetilde{G}_{n}$, then $I \in G_{n}$. The second statement follows immediately from Proposition 7.

Thus, although ${ }^{*} F_{3} \times \llbracket G \rrbracket$ is formally a group, $\llbracket \pi \rrbracket_{1}\left(\mathscr{L}_{\infty}, 0, \mathrm{f}\right)$ is not a group with respect to the groupoid structure defined by its action on $\llbracket \mathscr{L}_{\infty} \rrbracket$. It has nevertheless a distinguished subgroup isomorphic to ${ }^{*} F_{3}$. On the other hand,

TheOrem 9. Any two elements $\bullet v$ and $\cdot w$ of $\llbracket \pi \rrbracket_{1}\left(\mathscr{L}_{\infty}, 0, \mathrm{f}\right)$ define composable elements of the mother germ $\llbracket \pi \rrbracket_{1}\left(\mathscr{L}_{\infty}\right)$ by restriction of domains.

Proof. Let $I^{*} v, I_{*} w$ be the $\llbracket G \rrbracket$-coordinates of $\bullet v, \cdot w$. If ${ }^{*} v,{ }^{*} w$ and ${ }^{*} v+{ }^{*} w$ belong to ${ }^{*} V_{\infty}$ then by restricting $\bullet w$ to the leaf $S_{\infty}$ and restricting - $v$ to the leaf of $\llbracket \overline{\mathscr{L}}_{\infty} \rrbracket$ containing $\cdot w$, we obtain composable elements of $\llbracket \pi \rrbracket_{1}\left(\mathscr{L}_{\infty}\right)$. The other cases are handled similarly and are left to the reader.

The lamination $\mathscr{L}_{\infty}$ has the following property: every leaf $L \neq S_{\infty}$ is conformal to either $\mathbb{C}$ or $\mathbb{C}^{*}=\mathbb{C} \backslash\{(0,0)\}$, [6]. Hence $\mathscr{L}_{\infty}$ is neither a suspension nor a locally free action of a Lie group. In particular, the antenna lamination is beyond the purview of the definition of $\llbracket \pi \rrbracket_{1}$ found in $[4]$.

Given any leaf $L$ of $\mathscr{L}_{\infty}$, one can obtain a graphical model $T$ of $L$ as a limit of a sequence of translations of $T_{\infty}$. One can then repeat the discussion leading up to Theorem 8 for $L$. The proof of the following is left to the reader.

TheOREM 10. Let $L \subset \mathscr{L}_{\infty}$ be any leaf, modelled as above on a graph $T \in \mathbb{A}$ with vertex set $V$. Then for $v \in V$ and $\mathrm{f}$ constructed using a vector field as above, $\llbracket \pi \rrbracket_{1}\left(\mathscr{L}_{\infty}, v, \mathrm{f}\right)$ may be identified with

$$
{ }^{*} \pi_{1} L \times \llbracket G \rrbracket,
$$

where ${ }^{*} \pi_{1} L \times\{0\}$ is a subgroup with respect to the groupoid structure that $i s \cong 1$ or $* \mathbb{Z}$.

Corollary 3. Let $v \in V_{\infty} \subset S_{\infty}$ and $v^{\prime} \in V^{\prime} \subset L^{\prime} \neq S_{\infty}$. Then choosing frame fields as above, the fundamental germs $\llbracket \pi \rrbracket_{1}\left(\mathscr{L}_{\infty}, v, \mathrm{f}\right)$ and $\llbracket \pi \rrbracket_{1}\left(\mathscr{L}_{\infty}, v^{\prime}, \mathrm{f}^{\prime}\right)$ are not isomorphic. 
Proof. $\llbracket \pi \rrbracket_{1}\left(\mathscr{L}_{\infty}, v, \mathrm{f}\right)$ has a nonabelian subgroup whereas $\llbracket \pi \rrbracket_{1}\left(\mathscr{L}_{\infty}\right.$, $\left.v^{\prime}, f^{\prime}\right)$ is an abelian groupoid.

\section{§8. The $P S L(2, \mathbb{Z})$ Anosov foliation}

Let $\Gamma \subset P S L(2, \mathbb{R})$ be a discrete group of finite type, possibly with elliptic elements. The quotient $\Sigma=\mathbb{H}^{2} / \Gamma$ is a finite volume hyperbolic surface orbifold. The unit tangent bundle $T_{1} \Sigma$ is defined to be the quotient $\mathrm{T}_{1} \mathbb{H}^{2} / \Gamma$. Let $\rho: \Gamma \rightarrow$ Homeo $\left(\mathbb{S}^{1}\right)$ be the representation obtained by extending the action of $\Gamma$ to the boundary of $\mathbb{H}^{2}$. Then $T_{1} \Sigma$ may be identified with the suspension

$$
\left(\mathbb{H}^{2} \times \mathbb{S}^{1}\right) / \Gamma
$$

as follows. Given $(\tilde{z}, t) \in \mathbb{H}^{2} \times \mathbb{S}^{1}$, associate $\mathrm{v}_{\tilde{z}} \in \mathrm{T}_{1} \mathbb{H}^{2}$, the vector based at $\tilde{z}$ and tangent to the ray limiting to $t$. This association is $\Gamma$-equivariant and descends to the desired homeomorphism. The expression of $\mathrm{T}_{1} \Sigma$ as a suspension defines a hyperbolic Riemann surface foliation $\mathscr{F}$ on $\mathrm{T}_{1} \Sigma$, which is also a fiber bundle over $\Sigma$ provided that $\Gamma$ has no elliptic points. $\mathscr{F}$ is called an Anosov foliation.

In [4], we worked with a definition of $\llbracket \pi \rrbracket_{1}$ that was available for suspensions such as $\mathscr{F}$ formed from fixed point free $\Gamma$. Unfortunately, this hypothesis excluded the most "explicit" of discrete subgroups of $P S L(2, \mathbb{R})$, the modular group $\Gamma=P S L(2, \mathbb{Z})$. The definition provided in this paper is clearly available in this case, and we devote the rest of this section to its consideration.

Two elements $r, s \in \mathbb{R} \cup\{\infty\} \approx \mathbb{S}^{1}$ are called equivalent if there exists $A \in P S L(2, \mathbb{Z})$ such that $A(r)=s$. Every equivalence class $[r]$ of extended reals corresponds to a leaf $L_{[r]}$ of $\mathscr{F}$, and since all $\operatorname{PSL}(2, \mathbb{Z})$-orbits in $\mathbb{S}^{1}$ are dense, all leaves are dense. If $L_{[r]}$ is isomorphic to the punctured hyperbolic disk $\mathbb{D}^{*}$, then $[r]$ is quadratic over $\mathbb{Q}$. Otherwise, $L_{[r]}$ is isomorphic to $\mathbb{H}^{2}$.

Let us consider the leaf $L=L_{[0]} \cong \mathbb{D}^{*}$ covered by $\mathbb{H} \times\{0\}$. Choose $x \in L$ and a transversal $T$ through $x$ that is a fiber with respect to the projection onto the modular surface $\Sigma$. We assume that the lift $\tilde{x}$ of $x$ to $\mathbb{H}^{2}$ is not an elliptic point for the action of $\operatorname{PSL}(2, \mathbb{Z})$. Define $f$ to be the lift of a frame on $\Sigma$ based at the projection of $T$. As before, we denote by $\tilde{\mathrm{f}}$ the lift of $\mathrm{f}$ to $\widetilde{T}_{0} \subset \mathbb{H}^{2}$ and by $\tilde{\mathrm{f}}_{\tilde{y}}$ its value at $\tilde{y} \in \widetilde{T}_{0}$. Note that for $A \in P S L(2, \mathbb{R}), A_{*} \tilde{\mathrm{f}}_{\tilde{x}}=A_{*} \tilde{\tilde{f}}_{\hat{y}}$ if and only if $A \in \Gamma=P S L(2, \mathbb{Z})$. A sequence $\left\{A_{\alpha}\right\} \subset P S L(2, \mathbb{Z})$ defines an $\mathrm{f}$-diophantine approximation $\Leftrightarrow$ $\left(A_{\alpha} \tilde{x}, 0\right)$ projects to a sequence in $T$ converging to $x \Leftrightarrow\left(\tilde{x}, A_{\alpha}^{-1}(0)\right)$ projects 
to a sequence in $T$ converging to $x \Leftrightarrow A_{\alpha}^{-1}(0) \rightarrow 0$ in $\mathbb{S}^{1}$. Note that for any sequence $\left\{\gamma_{n_{\alpha}}\right\}$ in the deck group of $\mathbb{H}^{2} \rightarrow L$,

$$
\left\{\gamma_{n}=\left(\begin{array}{ll}
1 & 0 \\
n & 1
\end{array}\right) \mid n \in \mathbb{Z}\right\},
$$

the sequence $\left\{\gamma_{\alpha} A_{\alpha}\right\}$ also defines an $\mathrm{f}$-diophantine approximation. The fundamental germ $\llbracket \pi \rrbracket_{1}(\mathscr{F}, x, \mathrm{f})$ is then formed from the associated sequences $\left\{A_{\alpha} B_{\alpha}^{-1}\right\}$ where $\left\{B_{\alpha}\right\}$ is another $\mathrm{f}$-diophantine approximation.

Note 3. Writing

$$
A_{\alpha}=\left(\begin{array}{cc}
a_{\alpha} & b_{\alpha} \\
c_{\alpha} & d_{\alpha}
\end{array}\right)
$$

the sequences $\left\{-b_{\alpha} / a_{\alpha}=A_{\alpha}^{-1}(0)\right\}$ are hyperbolic diophantine approximations, as defined for example in [1]. See [4] for more on this point. In the case at hand, they give bad diophantine approximations of 0 whenever $b_{\alpha} \rightarrow \infty$, in the sense that it is never true that for some $c>0$ and almost all $\alpha$,

$$
\left|0-\left(-\frac{b_{\alpha}}{a_{\alpha}}\right)\right|<\frac{c}{a_{\alpha}^{2}} .
$$

The f-diophantine approximations are not stable with respect to the operation of inversion. Indeed, let $r \in \mathbb{R}$ be any real number, $\left\{m_{\alpha} / n_{\alpha}\right\}$ a sequence of rationals (written in lowest terms) converging to $r$. Let $M_{\alpha}, N_{\alpha}$ be such that $m_{\alpha} M_{\alpha}-n_{\alpha} N_{\alpha}=1$. Assume that the indexing is such that $\alpha+N_{\alpha} / m_{\alpha} \rightarrow \infty$ as $\alpha \rightarrow \infty$. Then the sequence $\left\{X_{\alpha}\right\}$,

$$
X_{\alpha}=\left(\begin{array}{cc}
-\left(\alpha m_{\alpha}+N_{\alpha}\right) & -m_{\alpha} \\
\alpha n_{\alpha}+M_{\alpha} & n_{\alpha}
\end{array}\right) \in P S L(2, \mathbb{Z}),
$$

satisfies $X_{\alpha}^{-1}(0) \rightarrow 0$, but $X_{\alpha}(0) \rightarrow-r$. Using this fact, we can now show

TheOREM 11. As a set,

$$
\llbracket \pi \rrbracket_{1}(\mathscr{F}, x, \mathrm{f})=P S L\left(2,{ }^{*} \mathbb{Z}\right) .
$$

Proof. Let $\left\{A_{\alpha}\right\}$ be any sequence in $P S L\left(2,{ }^{*} \mathbb{Z}\right)$. Then after passing to a subsequence if necessary, we find $A_{\alpha}^{-1}(0) \rightarrow r$ for some $r \in \mathbb{R} \cup\{\infty\}$. Note that $r$ is independent of the class of $\left\{A_{\alpha}\right\}$ in $P S L\left(2,{ }^{*} \mathbb{Z}\right)$. We may choose $\left\{n_{\alpha}\right\} \subset \mathbb{Z}$ so that $\gamma_{n_{\alpha}} A_{\alpha}^{-1}(0) \rightarrow 0$. Hence

$$
\left\{A_{\alpha}\right\}=\left\{A_{\alpha} \gamma_{-n_{\alpha}}\right\} \cdot\left\{\gamma_{-n_{\alpha}}^{-1}\right\}
$$

defines an element of $\llbracket \pi \rrbracket_{1}(\mathscr{F}, x, \mathrm{f})$. 
It is not difficult to see that with respect to its action on the germ universal cover $\llbracket \widetilde{\mathscr{F}} \rrbracket, \llbracket \pi \rrbracket_{1}(\mathscr{F}, x, \mathrm{f})$ is not a group. Indeed, the class of the sequence $\left\{X_{\alpha}^{-1}\right\}$, where $\left\{X_{\alpha}\right\}$ is the sequence appearing in (5), is not defined on $\widetilde{L}$.

\section{§. Mapping class group of the algebraic universal cover of a sur- face}

In this section, we use the fundamental germ to prove a Nielsen type theorem for the algebraic universal cover of a closed surface. We begin by recalling a few facts, referring the reader to [5] for details.

Let $\Sigma$ be a closed surface and let $\mathscr{G}=\left\{G_{\alpha}\right\}$ be the set of all normal finite index subgroups. For each $G_{\alpha}$, there exists a covering $\sigma_{\alpha}: \Sigma_{\alpha} \rightarrow \Sigma$ defined by the condition that $\pi_{1} \Sigma_{\alpha}$ maps isomorphically onto $G_{\alpha}$. If $G_{\alpha} \subset G_{\beta}$, there is a unique covering $s_{\alpha \beta}: \Sigma_{\alpha} \rightarrow \Sigma_{\beta}$ for which $\sigma_{\alpha}=\sigma_{\beta} \circ s_{\alpha \beta}$. Hence the collection of $\sigma_{\alpha}$ and $s_{\alpha \beta}$ forms an inverse system of surfaces by covering maps.

Definition 6. The algebraic universal cover of $\Sigma$ is the inverse limit

$$
\widehat{\Sigma}=\lim _{\longleftarrow} \Sigma_{\alpha} .
$$

If $\sigma: Z \rightarrow \Sigma$ is any finite covering, then $\sigma$ lifts to a homeomorphism

$$
\hat{\sigma}: \widehat{Z} \longrightarrow \widehat{\Sigma} \text {. }
$$

Thus the algebraic universal cover depends only on the type of $\Sigma$ (elliptic, parabolic, hyperbolic). In fact, there are only two non-trivial examples of algebraic universal covers of closed surfaces: that of the torus and that of a surface of hyperbolic type.

The inverse limit

$$
\hat{\pi}_{1} \Sigma=\lim _{\longleftarrow}\left(\pi_{1} \Sigma\right) / G_{\alpha}
$$

is a Cantor group called the profinite completion of $\pi_{1} \Sigma$. The homomorphism $i: \pi_{1} \Sigma \rightarrow \hat{\pi}_{1} \Sigma$ induced by the system of projections $\pi_{1} \Sigma \rightarrow \pi_{1} \Sigma / G_{\alpha}$ has dense image. Define a representation

$$
\begin{gathered}
\varsigma: \pi_{1} \Sigma \longrightarrow \operatorname{Homeo}\left(\hat{\pi}_{1} \Sigma\right) \\
\varsigma_{\gamma}(\hat{g})=\hat{g} \cdot i(\gamma)^{-1}
\end{gathered}
$$


for $\gamma \in \pi_{1} \Sigma$ and $\hat{g} \in \hat{\pi}_{1} \Sigma$. Then we may identify $\widehat{\Sigma}$ with the suspension of $\varsigma$ :

$$
\widehat{\Sigma} \approx\left(\widetilde{\Sigma} \times \hat{\pi}_{1} \Sigma\right) / \pi_{1} \Sigma
$$

With this identification, we see that $\widehat{\Sigma}$ is a surface lamination with Cantor transversals homeomorphic to $\hat{\pi}_{1} \Sigma$, that is, a solenoid. Moreover, it can also be seen from this presentation that every leaf $L$ of $\widehat{\Sigma}$ satisfies

$$
\pi_{1} L \cong \bigcap G_{\alpha}
$$

However for closed surfaces, $\bigcap G_{\alpha}=1$, so here, $L$ is simply connected. Each leaf $L$ is dense and a path-component of $\widehat{\Sigma}$. For every $\alpha$, the preimage of the projection map $\widehat{\Sigma} \rightarrow \Sigma_{\alpha}$ is a fiber transversal, homeomorphic to $\hat{\pi}_{1} \Sigma_{\alpha} \cong \widehat{G}_{\alpha}$.

Now let $L$ be a fixed leaf of $\widehat{\Sigma}$.

DEFINITION 7. The leafed mapping class group of $\widehat{\Sigma}$ is

$$
\operatorname{MCG}(\widehat{\Sigma}, L)=\operatorname{Homeo}(\widehat{\Sigma}, L) / \simeq
$$

where $\simeq$ is the relation of homotopy of homeomorphisms.

We denote by $[h]$ the mapping class associated to a homeomorphism $h$.

Let $G$ be a group.

DEFINITION 8. The virtual automorphism group of $G$ is

$$
\begin{aligned}
& \operatorname{Vaut}(G)=\left\{\phi: H \rightarrow H^{\prime} \mid \phi\right. \text { an isomorphism and } \\
& \left.\qquad H, H^{\prime} \text { finite index subgroups of } G\right\} / \sim,
\end{aligned}
$$

where $\phi_{1} \sim \phi_{2}$ if there exists $H^{\prime \prime}<G$ of finite index, contained in $\operatorname{Dom}\left(\phi_{1}\right) \cap$ $\operatorname{Dom}\left(\phi_{2}\right)$ and such that $\left.\phi_{1}\right|_{H^{\prime \prime}}=\left.\phi_{2}\right|_{H^{\prime \prime}}$.

Note that the equivalence relation $\sim$ is precisely what is needed to make composition of virtual automorphisms well-defined. We point out also that if $H<G$ is of finite index, then $\operatorname{Vaut}(H) \cong \operatorname{Vaut}(G)$. The following theorem is due to C. Odden [8]. We shall give a new proof using the mother germ and germ covering space theory.

TheOREM 12. $\operatorname{MCG}(\widehat{\Sigma}, L) \cong \operatorname{Vaut}\left(\pi_{1} \Sigma\right)$. 
Proof. Define a homomorphism

$$
\Theta: \operatorname{Vaut}\left(\pi_{1} \Sigma\right) \longrightarrow \operatorname{MCG}(\widehat{\Sigma}, L)
$$

as follows. Given $\phi: G_{1} \rightarrow G_{2}$ an isomorphism of finite index subgroups of $\pi_{1} \Sigma$, we may find covers $\sigma_{1}, \sigma_{2}: \Sigma^{\prime} \rightarrow \Sigma-$ indexed by $G_{1}$ and $G_{2}$ - so that

$$
\left(\sigma_{2}\right)_{*} \circ\left(\sigma_{1}\right)_{*}^{-1}=\phi:
$$

this follows from the classical Nielsen theorem. Then we define

$$
\Theta(\phi)=\left[\hat{\sigma}_{2} \circ \hat{\sigma}_{1}^{-1}\right]
$$

where for $i=1,2, \hat{\sigma}_{i}: \widehat{\Sigma}^{\prime} \rightarrow \widehat{\Sigma}$ is the algebraic universal cover lift. If $G^{\prime}<\operatorname{Dom}(\phi)$, then $\Theta\left(\left.\phi\right|_{G^{\prime}}\right)=\Theta(\phi)$, since $\Theta\left(\left.\phi\right|_{G^{\prime}}\right)$ is defined by the pair $\sigma_{i} \circ \sigma, i=1,2$, where $\sigma: \Sigma^{\prime \prime} \rightarrow \Sigma^{\prime}$ is a cover for which $\sigma_{1} \circ \sigma$ is indexed by $G^{\prime}$. Thus $\Theta$ is a well-defined homomorphism.

Claim 1. $\Theta$ is onto.

Let $h:(\widehat{\Sigma}, L) \rightarrow(\widehat{\Sigma}, L)$ be a homeomorphism. After performing an isotopy, we may arrange that $h$ fixes a point $x$ and fiber transversal $T$ containing $x$. Without loss of generality, we may assume that $T$ is a fiber transversal over $\Sigma$. Due to the suspension structure, $T \approx \hat{\pi} \Sigma$ : fix this identification so that $x \mapsto 1$ and $L \cap T \mapsto \pi_{1} \Sigma$. Since $h(L)=L$, we obtain a bijection

$$
h_{*}: \pi_{1} \Sigma \longrightarrow \pi_{1} \Sigma
$$

in which $h(1)=1$.

Suppose that for each $G_{\alpha}<\pi_{1} \Sigma, h_{*} \mid G_{\alpha}$ is not homomorphic. This means that for every $\alpha$, there exists $\gamma_{\alpha}, \gamma_{\alpha}^{\prime} \in G_{\alpha}$ so that

$$
h_{*}\left(\gamma_{\alpha} \cdot \gamma_{\alpha}^{\prime}\right) \neq h_{*}\left(\gamma_{\alpha}\right) \cdot h_{*}\left(\gamma_{\alpha}^{\prime}\right) \text {. }
$$

Assuming that $\widehat{\Sigma}$ has been equipped with a hyperbolic metric, say lifted from $\Sigma$, then the sequences $\left\{\gamma_{\alpha}\right\},\left\{\gamma_{\alpha}^{\prime}\right\}$ define elements of the fundamental germ

$$
{ }^{*} \gamma,{ }^{*} \gamma^{\prime} \in \llbracket \pi \rrbracket_{1}(\widehat{\Sigma}, x, \mathrm{f})
$$

where $f$ is a frame field lifted from a frame on $\Sigma$. But this fundamental germ is a subgroup of the mother germ $\llbracket \pi \rrbracket_{1}(\widehat{\Sigma})$. By Theorem $7, h$ induces a groupoid isomorphism

$$
\llbracket h \rrbracket_{*}: \llbracket \pi \rrbracket_{1}(\widehat{\Sigma}) \longrightarrow \llbracket \pi \rrbracket_{1}(\widehat{\Sigma}),
$$


and so we must have

$$
\llbracket h \rrbracket_{*}\left({ }^{*} \gamma \cdot{ }^{*} \gamma^{\prime}\right)=\llbracket h \rrbracket_{*}\left({ }^{*} \gamma\right) \cdot \llbracket h \rrbracket_{*}\left({ }^{*} \gamma^{\prime}\right)
$$

This contradicts equation (6). Thus $h_{*}$ defines an isomorphism when restricted to some $G_{\alpha}$, and this isomorphism determines an element $\phi \in$ $\operatorname{Vaut}\left(\pi_{1} \Sigma\right)$. Note that that $\phi$ does not depend on the isotopy used to ensure $h(x)=x$ since the holonomy group of $\widehat{\Sigma}$ at any point is trivial. Choose $\sigma_{i}: \Sigma^{\prime} \rightarrow \Sigma, i=1,2$, so that $\Theta(\phi)=\left[\hat{\sigma}_{2} \circ \hat{\sigma}_{1}^{-1}\right]$. To simplify notation, we write $h_{0}=\hat{\sigma}_{2} \circ \hat{\sigma}_{1}^{-1}$.

Recall that since $\widehat{\Sigma}$ is compact with hyperbolic leaves, the germ universal cover of $\widehat{\Sigma}$ is $\bullet \mathbb{H}^{2}$. The homeomorphisms $h$ and $h_{0}$ lift to the standard bijections $\bullet$ and $\bullet h_{0}$ of $\bullet \mathbb{H}^{2}$ sharing the same equivariance with respect to the action of the mother germ $\llbracket \pi \rrbracket_{1}(\widehat{\Sigma})$. In particular, they act identically on the set of galaxies of $\bullet \mathbb{H}^{2}$. For this reason, we may choose a germ universal cover topology for $\bullet^{2}$ with respect to which both $\bullet h$ and $\bullet h_{0}$ are homeomorphisms.

Define a homotopy $\boldsymbol{H}_{t}$ from $\bullet \boldsymbol{h}$ to $\boldsymbol{h}_{0}$ as follows. For each $\boldsymbol{}^{z} \in \boldsymbol{\bullet}^{\mathbf{2}}$, $\bullet H_{t}(\bullet z)$ is the point subdividing the hyperbolic geodesic connecting $\bullet h(\bullet z)$ to $h_{0}(\bullet z)$ into the proportion $t: 1-t$. By construction, ${ }^{\bullet} H_{t}$ has the same equivariance as $\bullet$ and $\bullet h_{0}$ and is in particular continuous. Since its initial and final maps are standard, so is $\bullet H_{t}$. By Proposition 6 , it descends to a homotopy $H_{t}$ of $h$ and $h_{0}$. It follows that $[h]=\left[h_{0}\right]=\Theta(\phi)$, and $\Theta$ is onto.

\section{Claim 2. $\Theta$ is one-to-one.}

If not, then there exists $\phi \neq$ the identity map with $\Theta(\phi)=1$. But then $\Theta(\phi)$ would have to induce the identity map on the mother germ; by construction, this can only happen if $\phi$ is trivial.

Let $\operatorname{Mod}(\widehat{\Sigma}, L)$ be the Teichmüller modular group of the pair $(\widehat{\Sigma}, L)$ : the group of homotopy classes of quasiconformal homeomorphisms of $\widehat{\Sigma}$ that preserve $L$.

\section{Corollary 4. $\operatorname{Mod}(\widehat{\Sigma}, L)=\operatorname{MCG}(\widehat{\Sigma}, L)$.}

Proof. This follows from the proof of Theorem 12 and the fact that every finite cover of compact Riemann surfaces is homotopic to a quasiconformal cover. 
Theorem 12 can be used to formulate the following conjectural Nielsentype theorem. Given $x \in L$, the fundamental germ $\llbracket \pi \rrbracket_{1}(\widehat{\Sigma}, x)$ is made up of all sequences $\left\{\gamma_{\alpha}\right\}$ converging with respect to the lattice of finite index normal subgroups $G$ of $\pi_{1} \Sigma$, so

$$
\llbracket \pi \rrbracket_{1}(\widehat{\Sigma}, x) \cong \bigcap_{\left[\pi_{1} \Sigma: G\right]<\infty}{ }^{*} G \subset{ }^{*} \pi_{1} \Sigma .
$$

It follows then that there is a monomorphism $\operatorname{Vaut}\left(\pi_{1}\right) \hookrightarrow \operatorname{Aut}\left(\llbracket \pi \rrbracket_{1}(\widehat{\Sigma}, x)\right)$, which descends to $\operatorname{Vaut}\left(\pi_{1}\right) \hookrightarrow \operatorname{Out}\left(\llbracket \pi \rrbracket_{1}(\widehat{\Sigma}, x)\right)$ upon passage to the quotient. This latter map is also a monomorphism, since no nontrivial virtual automorphism $\phi$ can induce on $\llbracket \pi \rrbracket_{1}(\widehat{\Sigma}, x)$ an inner automorphism. For otherwise, $\phi$ would have to be inner on some subgroup $H$, hence all, which is only possible if $\phi$ is trivial. In view of these remarks we

ConjeCture. The monomorphism $\operatorname{MCG}(\widehat{\Sigma}, L) \hookrightarrow \operatorname{Out}\left(\llbracket \pi \rrbracket_{1}(\widehat{\Sigma}, x)\right)$ is an isomorphism.

We end this section by explaining the importance of Theorem 12 and Corollary 4 in giving a genus independent reformulation the Ehrenpreis conjecture. The classical Ehrenpreis conjecture is:

Given two closed hyperbolic surfaces $\Sigma_{1}$ and $\Sigma_{2}$ and $\epsilon>0$, there exist finite, locally isometric covering surfaces $Z_{1}$ and $Z_{2}$ of each which are $(1+\epsilon)$-quasiisometric.

We then have the following equivalent, genus independent version:

Every orbit of the action of $\operatorname{Mod}(\widehat{\Sigma}, L)$ on $\mathscr{T}(\widehat{\Sigma})$ is dense.

In other words, the genus independent version says that, although the moduli space

$$
\mathscr{T}(\widehat{\Sigma}) / \operatorname{Mod}(\widehat{\Sigma}, L)
$$

is uncountable, it has the "topology of a point" (i.e. the coarse topology). If affirmed, the Ehrenpreis conjecture would thus provide an explanation for the jump between the existence of moduli (dimension 2) and rigidity (dimension 3 and higher) in hyperbolic geometry. See the articles [2], [5] for more discussion. 


\section{REFERENCES}

[1] V. I. Bernik and M. M. Dodson, Metric Diophantine Approximation on Manifolds, Cambridge Tracts in Mathematics 137, Cambridge University Press, Cambridge, 1999.

[2] I. Biswas, S. Nag and D. P. Sullivan, Determinant bundles, Quillen metrics and Mumford isomorphisms over the universal commensurability Teichmüller space, Acta Math., 176 (1996), no. 2, 145-169.

[3] T. M. Gendron, Fuchsian Germs, PhD Thesis, City University of New York, 1997.

[4] T. M. Gendron, The algebraic theory of the fundamental germ, Bull. Braz. Math. Soc. (N.S.), 37 (2006), no. 1, 49-87.

[5] T. M. Gendron, The Ehrenpreis conjecture and the moduli-rigidity gap, Complex Manifolds and Hyperbolic Geometry (Earle et al, eds.), Contemporary Mathematics, AMS, Providence, RI, 2002, pp. 207-229.

[6] E. Ghys, Laminations par surfaces de Riemann, Dynamique et géométrie complexes (Lyon, 1997), Panoramas \& Synthèse 8, Soc. Math. France, Paris, 1999, pp. 49-95.

[7] R. Goldbladt, Lectures on the Hyperreals, Springer-Verlag, New York, NY, 1998.

[8] C. Odden, The baseleaf preserving mapping class group of the universal hyperbolic solenoid, Trans. Amer. Math. Soc., 357 (2005), no. 5, 1829-1858.

[9] A. Robinson, Non-standard Analysis, Princeton Landmarks in Mathematics, Princeton University Press, Princeton, NJ, 1996.

[10] K. D. Stroyan and W. A. J. Luxemburg, Introduction to the theory of infinitesimals, Pure and Applied Mathematics, No. 72, Academic Press, New York-London, 1976.

Instituto de Matemáticas - Unidad Cuernavaca

Universidad Nacional Autónoma de México

Av. Universidad $s / n$

C.P. 62210 Cuernavaca, Morelos

$M E ́ X I C O$

tim@matcuer.unam.mx 